\title{
QUEEN'S
UNIVERSITY
BELFAST
}

\section{Transmission Scheme and Performance Analysis of Multi-Cell Decoupled Heterogeneous Networks}

Liu, W., Jin, S., Matthaiou, M., \& You, X. (2020). Transmission Scheme and Performance Analysis of Multi-Cell Decoupled Heterogeneous Networks. IEEE Transactions on Communications.

https://doi.org/10.1109/TCOMM.2020.2988283

Published in:

IEEE Transactions on Communications

Document Version:

Peer reviewed version

Queen's University Belfast - Research Portal:

Link to publication record in Queen's University Belfast Research Portal

Publisher rights

Copyright 2020 IEEE. This work is made available online in accordance with the publisher's policies. Please refer to any applicable terms of use of the publisher.

\section{General rights}

Copyright for the publications made accessible via the Queen's University Belfast Research Portal is retained by the author(s) and / or other copyright owners and it is a condition of accessing these publications that users recognise and abide by the legal requirements associated with these rights.

Take down policy

The Research Portal is Queen's institutional repository that provides access to Queen's research output. Every effort has been made to ensure that content in the Research Portal does not infringe any person's rights, or applicable UK laws. If you discover content in the Research Portal that you believe breaches copyright or violates any law, please contact openaccess@qub.ac.uk. 


\title{
Transmission Scheme and Performance Analysis of Multi-Cell Decoupled Heterogeneous Networks
}

\author{
Wen Liu, Student Member, IEEE, Shi Jin, Senior Member, IEEE, \\ Michail Matthaiou, Senior Member, IEEE and Xiaohu You, Fellow, IEEE
}

\begin{abstract}
Although uplink (UL) downlink (DL) decoupling (DUDe) brings significant gains in the UL throughput of decoupled user equipments (DeUEs) in heterogeneous networks, channel estimation and DL performance of DeUEs are worse than the coupled UEs due to the DUDe property and the cell edge effect. To address these fundamental problems, we propose a transmission scheme with data-aided (DA) minimum mean square error (MMSE) channel estimator and zero-forcing (ZF) interference nulling (IN) precoding for a two-tier multi-cell HetNet with DUDe. We first present a method to estimate the bit error rate (BER) of UL data, then, derive the form of DA MMSE estimator, which utilizes decoded UL data, estimated BER and known UL training sequences to jointly estimate the DL channels of DeUEs. ZF IN precoding uses the estimated channels of DeUEs to cancel the nearest DL interference without any cooperation and message transmission. Also, we derive a tight approximation to the achievable DL rate of DeUEs and analyze the benefits of the DA estimator and ZF IN precoding. Our simulations show that DA MMSE estimator outperforms the conventional MMSE counterpart, while the proposed scheme improves the DL performance of both DeUEs and macro UEs, though the rate gain may be degraded by pilot contamination and inter-cell interference.
\end{abstract}

Index Terms-Data-aided channel estimation, decoupled heterogeneous networks, downlink performance, pilot contamination, interference-nulling.

\section{INTRODUCTION}

To realize the requirements of $5 \mathrm{G}$ systems with higher data traffic and lower energy consumption [1], deploying massive multiple-input multiple-output (MIMO) base stations (BSs) in heterogeneous networks (HetNets) is regarded as a promising solution [2]-[4]. In this way, massive MIMO BSs can significantly improve the capacity and reliability while low-cost small BSs (SBSs) can provide high throughput and dense coverage over a small range [5]. Although massive MIMO works well with HetNets, some major changes are required to the existing network architectures and transmission schemes, particularly in terms of interference management [6],

The work of W. Liu and X. You was supported by the National Natural Science Foundation of China (NSFC) under Grant 61521061. The work of S. Jin was supported by the NSFC under Grant 61531011. The work of M. Matthaiou was supported by a research grant from the Department for the Economy Northern Ireland under the US-Ireland R\&D Partnership Programme and by the EPSRC, U.K., under Grant EP/P000673/1.

W. Liu, S. Jin and X. You are with the National Mobile Communications Research Laboratory, Southeast University, Nanjing 210096, China (e-mail: newen@seu.edu.cn; jinshi@seu.edu.cn; xhyu@seu.edu.cn).

M. Matthaiou is with the Institute of Electronics, Communications and Information Technology (ECIT), Queen's University Belfast, Belfast BT3 9DT, U.K. (e-mail: m.matthaiou@qub.ac.uk). cell association [7] and resource allocation [8], to make the two technologies more synchronized.

Recently, a new trend in cell association of uplink (UL) downlink (DL) decoupling (DUDe) has been proposed by [9], which breaks the constraint that user equipments (UEs) must associate with the same BSs in UL/DL. Compared with the coupled solution, DUDe can bring significant gains in UL throughput, outage, and energy efficiency at a very low cost [10]. However, some open problems are still required to be addressed before the implementation of DUDe. Decoupled UEs (DeUEs) associate with different BSs in UL/DL, which causes the channel state information (CSI) acquisition problem since the estimated UL channel at one BS cannot be used for DL precoding at another BS [11]. Besides, when considering multi-cell cellular HetNets, pilot contamination will deteriorate the accuracy of the DeUEs' estimated channels. Moreover, DeUEs in a HetNet with DUDe can be recognized as celledge UEs in a regular cellular network because DeUEs are not close to either their UL BSs or DL BSs, otherwise, they tend to connect to the same BS in both ways. As a result, a DeUE cannot receive a strong desired signal from its DL BS while suffering severe interference from its UL BS. Thus, although the UL throughput of DeUEs can be improved significantly by DUDe, the DL performance can be rather poor compared with coupled UEs. Therefore, a transmission scheme for DeUEs is urgently needed to enhance the channel estimation and DL performance.

The previous research of DUDe in HetNets mainly focus on the UL performance; in this context, improvements in throughput and signal-to-interference-and-noise- ratio (SINR) have been confirmed by both simulations and theoretical analysis [10]. Also, [12] studied the UL and DL performance in decoupled HetNets with Sub-6G and mmWave BSs distributed as Poisson point processes (PPPs). Although the PPP model provides an analytic framework for rate coverage analysis, macro BSs (MBSs) modeled by PPP are distributed independently and two MBSs may be too close to each other. Thus, in this paper, MBSs are deployed in a regular way while SBSs are placed randomly. In most of these papers ( e.g., [9], [12]-[14]), the PPP model is adopted and perfect CSI is assumed at both MBSs and SBSs, however, imperfect CSI must be taken into account in decoupled HetNets, especially for DeUEs. Recall that accurate CSI is important for satisfactory DL performance in massive MIMO systems while pilot contamination due to pilot reuse creates another strong directional interference. Thus, several methods for contamination mitigation have been proposed, such as the time-shifted protocol in [15], covariance- 
aided channel estimation in [16] and pilot contamination precoding in [17]. Data-aided (DA) channel estimation in massive MIMO systems is studied in [18] where the estimated channel is updated iteratively with the sequentially decoded UL data. Finally, our prior work [11] developed a DA minimum mean square error (MMSE) estimator for single-cell HetNets to further exploit the channel information in UL data signal where the effects of pilot contamination and inter-cell interference were not considered.

In this paper, we focus on improving the DL performance of UEs in multi-cell decoupled HetNets and propose a novel transmission scheme, which applies the DA channel estimation and zero-forcing (ZF) interference-nulling (IN) precoding to mitigate pilot contamination and intra-cell interference. Also, we develop an analytical framework to analyze the normalized mean square error (NMSE) of DA channel estimator and DL throughput in a multi-cell decoupled HetNet. The specific contributions of this paper are summarized as follows:

- We develop a DA MMSE channel estimator for multicell decoupled HetNets, which utilizes decoded UL data sequences and estimated bit error rate (BER) values along with known UL training sequences to jointly estimate the DL channels of DeUEs at a MBS. Compared with the DA estimator in [11], the proposed estimator takes into account the pilot contamination and can acquire more accurate CSI than pilot-based estimators in multi-cell scenarios.

- To further boost the DL performance, we develop a new ZF IN precoding performed at UL BSs of DeUEs, which cancels the nearest interference of DeUEs by projecting the classic ZF precoding matrix into the nulling subspace spanned by the estimated channels of DeUEs. ZF IN precoding exploits the inherent benefit of DUDe structure, thus no cooperation and message exchange are required.

- We provide a method to estimate BER values of UL data and derive the NMSE expression of the multi-cell DA MMSE estimator. To investigate the effect of pilot contamination, NMSE performance of DA methods are compared with the conventional MMSE and least square (LS) estimators in both single and multi-cell scenarios. Additionally, we derive a closed-form approximation of the achievable DL rate of DeUEs, through which, we are able to learn analytically how the DL rate benefits from the DA methods and ZF IN precoding, and how the DL performance is affected by the system parameters.

The rest of the paper is organized as follows. Section II presents the system model and DUDe cell association. Section III describes the proposed 4-stage transmission scheme for decoupled HetNets. The theoretical analysis and discussion of DA MMSE estimator and the achievable DL rate are shown in Section IV. We provide numerical results in Section V and finally conclude the paper in Section VI.

Notation: Boldface lowercase and capital letters denote vectors and matrices; the Hermitian of the matrix $\mathbf{A}$ is denoted by $\mathbf{A}^{\mathrm{H}}$ and $[\mathbf{A}]_{i j}$ is the $(i, j)$-th entry of $\mathbf{A} ; \mathbf{0}_{M}$ is a $M \times 1$ zero vector and $\mathbf{I}_{M}$ is an $M \times M$ identity matrix; $\operatorname{diag}\left(a_{1} \ldots a_{K}\right)$ denotes the diagonal matrix with elements $a_{1} \ldots a_{K} . \mathbb{E}[\cdot]$ and

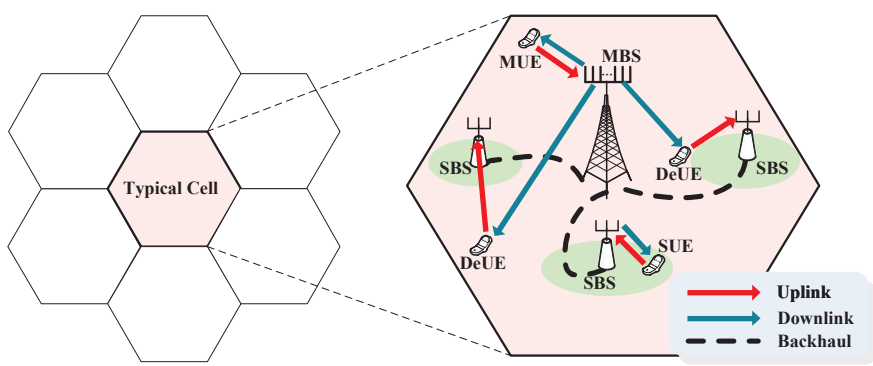

Fig. 1. Illustration of a two-tier multi-cell HetNet with decoupling access.

$\operatorname{Tr}[\cdot]$ denote the operators of expectation and trace; $\|\cdot\|_{2}$ and $|\cdot|$ denote the $\ell_{2}$-norm of a vector and the absolute value of a complex number, respectively. Finally, $\mathbf{z} \sim \mathcal{C N}\left(\mathbf{0}_{M}, \mathbf{R}_{M}\right)$ means $\mathbf{z}$ is a complex Gaussian random vector with zero mean and covariance matrix $\mathbf{R}_{M}$.

\section{SYSTEM MODEL}

We consider a multi-cell cellular HetNet with $L$ cells where each macro-cell has a MBS with $M$ co-located antennas in the cell center, $S$ SBSs with $N$ antennas and $K$ single-antenna UEs are randomly placed in the range of the cell, as depicted in Fig. 1. Note that in each cell, wired backhaul links are deployed between the central MBS and SBSs to collect decoded data with negligible error and latency [10]. We assume the whole system operates in time-division duplexing (TDD) mode and all BSs and UEs are in perfect synchronization. The total system frequencies are fully reused between cells, thus the model of this paper is pilot-contaminated and interferencelimited. The noise at all nodes is modeled as additive white Gaussian noise (AWGN) whose entries are i.i.d. $\mathcal{C N}\left(0, N_{0}\right)$ random variables, and $N_{0}$ is the noise power spectral density.

The channel between any two nodes is assumed to be frequency non-selective and follows independent block fading, which means channels are mutually independent and remain constant within each coherence block. By neglecting shadowing effect and differences between co-located antennas at the same BS [19], the channels between the $k$-th UE in the $i$-th cell to the MBS and the $v$-th SBS of cell $j$ are

$$
\begin{aligned}
\mathbf{h}_{i k j} & =\sqrt{\beta_{i k j}^{\mathrm{M}}} \mathbf{h}_{i k j}^{\mathrm{W}}, \\
\mathbf{g}_{i k j v} & =\sqrt{\beta_{i k j v}^{\mathrm{S}}} \mathbf{g}_{i k j v}^{\mathrm{W}},
\end{aligned}
$$

where $\mathbf{h}_{i k j}^{\mathrm{W}} \sim \mathcal{C N}\left(\mathbf{0}_{M}, \mathbf{I}_{M}\right)$ and $\mathbf{g}_{i k j v}^{\mathrm{W}} \sim \mathcal{C N}\left(\mathbf{0}_{N}, \mathbf{I}_{N}\right)$ model independent Rayleigh fading; $\beta_{i k j}^{\mathrm{M}}, \beta_{i k j v}^{\mathrm{S}}$ represent the largescale fading from a UE to a MBS and a SBS, respectively. Thus, the aggregate channel matrices of UEs in the $i$-th cell to the MBS and the $v$-th SBS of cell $j$ can be written as

$$
\begin{aligned}
\mathbf{H}_{i j} & =\mathbf{H}_{i j}^{\mathrm{W}} \mathbf{R}_{\mathbf{H}_{i j}, 2}^{1 / 2}, \\
\mathbf{G}_{i j v} & =\mathbf{G}_{i j v}^{\mathrm{W}} \mathbf{R}_{\mathbf{G}_{i j v}}^{1 / 2},
\end{aligned}
$$

where $\mathbf{H}_{i j}^{\mathrm{W}} \in \mathbb{C}^{M \times K}, \mathbf{G}_{i j v}^{\mathrm{W}} \in \mathbb{C}^{N \times K}$ are denoted as $\mathbf{H}_{i j}^{\mathrm{W}}=\left[\mathbf{h}_{i 1 j}^{\mathrm{W}} \ldots \mathbf{h}_{i K j}^{\mathrm{W}}\right]$ and $\mathbf{G}_{i j v}^{\mathrm{W}}=\left[\mathbf{g}_{i 1 j v}^{\mathrm{W}} \ldots \mathbf{g}_{i K j v}^{\mathrm{W}}\right] ; \mathbf{R}_{\mathbf{H}_{i j}}=$ $\operatorname{diag}\left(\beta_{i 1 j}^{\mathrm{M}} \ldots \beta_{i K j}^{\mathrm{M}}\right)$ and $\mathbf{R}_{\mathbf{G}_{i j v}}=\operatorname{diag}\left(\beta_{i 1 j v}^{\mathrm{S}} \ldots \beta_{i K j v}^{\mathrm{S}}\right)$ are $K \times K$ diagonal matrices.

Different from the conventional decoupled association policy based on the DL reference signal received power [9], we 


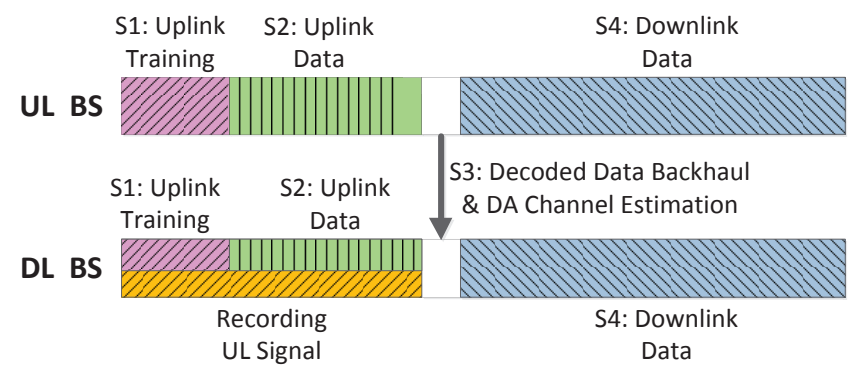

Fig. 2. Transmission frame structure of DeUEs in a decoupled HetNet involving DA channel estimation.

adopt the modified maximum average downlink receive power (MARP) policy introduced in [11] which considers the array gains of different types of BSs with multiple antennas. To be more specific, let $\mathrm{UE}_{l k}$ denote the $k$-th $\mathrm{UE}$ in cell $l$ and the associated BSs in UL/DL of $\mathrm{UE}_{l k}$ can be expressed as

$$
\begin{aligned}
\mathcal{U}_{l k} & =\underset{v=0,1, \ldots, S}{\arg \max }\left\{\mathbb{E}\left[Q_{l k l v}^{\mathrm{UL}}\right]\right\}, \\
\mathcal{D}_{l k} & =\underset{v=0,1, \ldots, S}{\arg \max }\left\{\mathbb{E}\left[Q_{l k l v}^{\mathrm{DL}}\right]\right\},
\end{aligned}
$$

and $\left[\mathcal{U}_{l k}\right], \quad\left[\mathcal{D}_{l k}\right],(l \in\{1, \ldots, L\}, k \in\{1, \ldots, K\})$ are the association matrices of $\mathrm{UL}$ and DL. $Q_{l k l v}^{\mathrm{UL}}$ and $Q_{l k l v}^{\mathrm{DL}}$ are defined as

$$
\begin{aligned}
Q_{l k l v}^{\mathrm{UL}} & = \begin{cases}P_{\mathrm{D}}\left\|\mathbf{h}_{l k l}\right\|_{2}^{2} & v=0, \\
P_{\mathrm{D}}\left\|\mathbf{g}_{l k l v}\right\|_{2}^{2} & v=1, \ldots, S,\end{cases} \\
Q_{l k l v}^{\mathrm{DL}} & = \begin{cases}P_{\mathrm{M}}\left\|\mathbf{h}_{l k l}\right\|_{2}^{2} & v=0, \\
P_{\mathrm{S}}\left\|\mathbf{g}_{l k l v}\right\|_{2}^{2} & v=1, \ldots, S,\end{cases}
\end{aligned}
$$

where $P_{\mathrm{D}}, P_{\mathrm{S}}$ and $P_{\mathrm{M}}$ are the transmit power of UEs, SBSs and MBSs, respectively. Thus, UEs are categorized into three types, which are MUEs, SUEs and DeUEs ${ }^{1}$, and

$$
\mathrm{UE}_{i k} \text { is } \begin{cases}\text { a MUE } & \mathcal{D}_{i k}=\mathcal{U}_{i k}=0, \\ \text { a SUE } & \mathcal{D}_{i k}=\mathcal{U}_{i k}=v, v=1, \ldots, S, \\ \text { a DeUE } & \mathcal{D}_{i k}=0, \mathcal{U}_{i k} \neq 0 .\end{cases}
$$

Such a cell association policy allows UEs to connect to their optimal BSs in UL/DL and enhances the UL throughput of decoupled UEs. However, this flexible association policy also creates the fundamental problem that how to acquire reliable CSI of decoupled UEs at their DL BSs. Moreover, pilot contamination makes CSI acquisition even more problematic. Therefore, a transmission scheme is proposed for decoupled HetNets to combat the impact of pilot contamination and, eventually, boost the DL performance.

\section{TRANSMISSION SCHEME}

In this section, we develop a transmission scheme for decoupled multi-cell HetNets by utilizing DA MMSE channel estimation and ZF IN precoding. As illustrated in Fig. 2, a new frame structure consisting of four stages is designed specifically for the proposed scheme. The detailed signal model and implementation of the scheme are presented as follows.

\footnotetext{
${ }^{1}$ Normally, we assume $P_{\mathrm{M}}>P_{\mathrm{S}}$ and $M>N$, such that UEs will not connect to an MBS in UL and an SBS in DL under our association policy.
}

\section{A. Uplink Training}

At the beginning of a frame, $K$ UEs in each cell send $K$ mutually orthogonal uplink training sequences, which are randomly assigned to UEs and fully reused within all cells; $\mathbf{s}_{k} \in \mathbb{C}^{1 \times \tau_{\mathrm{T}}}$ is the $k$-th orthogonal training sequence allocated to the $k$-th UE in each cell. By writing the training matrix as $\mathbf{S}=\left[\mathbf{s}_{1}^{\mathrm{H}} \ldots \mathbf{s}_{K}^{\mathrm{H}}\right]^{\mathrm{H}}$, we have $\mathbf{S S}^{\mathrm{H}}=\tau_{\mathrm{T}} P_{\mathrm{T}} \mathbf{I}_{K}$ when $\tau_{\mathrm{T}} \geqslant K$, $P_{\mathrm{T}}$ is the training power.

The received signals at the MBS and the $v$-th SBS in cell $i$ are expressed as

$$
\begin{aligned}
& \mathbf{Y}_{i 0}^{\mathrm{UT}}=\sum_{l=1}^{L} \mathbf{H}_{l i} \mathbf{S}+\mathbf{N}_{i 0}^{\mathrm{UT}}, \\
& \mathbf{Y}_{i v}^{\mathrm{UT}}=\sum_{l=1}^{L} \mathbf{G}_{l i v} \mathbf{S}+\mathbf{N}_{i v}^{\mathrm{UT}},
\end{aligned}
$$

where $\mathbf{N}_{i 0}^{\mathrm{UT}}, \mathbf{N}_{i v}^{\mathrm{UT}}$ are AWGNs at the MBS and the $v$-th SBS.

By utilizing the LS channel estimator [16], the channels of $\mathrm{UE}_{i k}$ to the $v$-th SBS and the MBS in cell $i$ are estimated as

$$
\begin{aligned}
\hat{\mathbf{h}}_{i k i}^{\mathrm{LS}} & =\sum_{l=1}^{L} \mathbf{h}_{l k i}+\frac{1}{\tau_{\mathrm{T}} P_{\mathrm{T}}} \mathbf{N}_{i 0}^{\mathrm{UT}} \mathbf{s}_{k}^{\mathrm{H}}, \\
\hat{\mathbf{g}}_{i k i v}^{\mathrm{LS}} & =\sum_{l=1}^{L} \mathbf{g}_{l k i v}+\frac{1}{\tau_{\mathrm{T}} P_{\mathrm{T}}} \mathbf{N}_{i v}^{\mathrm{UT}} \mathbf{s}_{k}^{\mathrm{H}} .
\end{aligned}
$$

The MMSE estimator can also be adopted to estimate the channels as [20]

$$
\begin{aligned}
\hat{\mathbf{h}}_{i k i}^{\mathrm{MMSE}} & =\frac{\beta_{i k i}^{\mathrm{M}} \rho^{\mathrm{PO}}}{1+\rho^{\mathrm{PO}} \sum_{l=1}^{L} \beta_{l k i}^{\mathrm{M}}}\left(\sum_{l=1}^{L} \mathbf{h}_{l k i}+\frac{1}{\tau_{\mathrm{T}} P_{\mathrm{T}}} \mathbf{N}_{i 0}^{\mathrm{UT}} \mathbf{s}_{k}^{\mathrm{H}}\right), \\
\hat{\mathbf{g}}_{i k i v}^{\mathrm{MMSE}} & =\frac{\beta_{i k i v}^{\mathrm{S}} \rho^{\mathrm{PO}}}{1+\rho^{\mathrm{PO}} \sum_{l=1}^{L} \beta_{l k i v}^{\mathrm{S}}}\left(\sum_{l=1}^{L} \mathbf{g}_{l k i v}+\frac{1}{\tau_{\mathrm{T}} P_{\mathrm{T}}} \mathbf{N}_{i v}^{\mathrm{UT}} \mathbf{s}_{k}^{\mathrm{H}}\right) .
\end{aligned}
$$

where $\rho^{\mathrm{PO}}=\frac{\tau_{\mathrm{T}} P_{\mathrm{T}}}{N_{0}}$. By defining the estimation error vectors as $\tilde{\mathbf{h}}_{i k i}^{\mathrm{MMSE}}=\mathbf{h}_{i k i}-\hat{\mathbf{h}}_{i k i}^{\mathrm{MMSE}}$ and $\tilde{\mathbf{g}}_{i k i v}^{\mathrm{MMSE}}=\mathbf{g}_{i k i v}-\hat{\mathbf{g}}_{i k i v}^{\mathrm{MMSE}}$, we know that the estimated channels and error vectors are mutually independent due to the property of MMSE estimator [20], thus we have $\hat{\mathbf{h}}_{i k i}^{\mathrm{MMSE}} \sim \mathcal{C N}\left(\mathbf{0}, \hat{\beta}_{i k i}^{\mathrm{M}} \mathbf{I}_{M}\right), \tilde{\mathbf{h}}_{i k i}^{\mathrm{MMSE}} \sim \mathcal{C N}\left(\mathbf{0}, \tilde{\beta}_{i k i}^{\mathrm{M}} \mathbf{I}_{M}\right)$, $\hat{\mathbf{g}}_{i k i v}^{\mathrm{MMSE}} \sim \mathcal{C N}\left(\mathbf{0}, \hat{\beta}_{i k i v}^{\mathrm{S}} \mathbf{I}_{M}\right), \tilde{\mathbf{g}}_{i k i v}^{\mathrm{MMSE}} \sim \mathcal{C N}\left(\mathbf{0}, \tilde{\beta}_{i k i v}^{\mathrm{S}} \mathbf{I}_{M}\right)$ with

$$
\begin{gathered}
\hat{\beta}_{i k i}^{\mathrm{M}}=\frac{\rho^{\mathrm{PO}}\left(\beta_{i k i}^{\mathrm{M}}\right)^{2}}{1+\rho^{\mathrm{PO}} \sum_{l=1}^{L} \beta_{l k i}^{\mathrm{M}}}, \quad \tilde{\beta}_{i k i}^{\mathrm{M}}=\frac{\beta_{i k i}^{\mathrm{M}}+\rho^{\mathrm{PO}} \beta_{i k i}^{\mathrm{M}} \sum_{l=1, l \neq i}^{L} \beta_{l k i}^{\mathrm{M}}}{1+\rho^{\mathrm{PO}} \sum_{l=1}^{L} \beta_{l k i}^{\mathrm{M}}}, \\
\hat{\beta}_{i k i v}^{\mathrm{S}}=\frac{\rho^{\mathrm{PO}}\left(\beta_{i k i v}^{\mathrm{S}}\right)^{2}}{1+\rho^{\mathrm{PO}} \sum_{l=1}^{L} \beta_{l k i v}^{\mathrm{S}}}, \quad \tilde{\beta}_{i k i v}^{\mathrm{S}}=\frac{\beta_{i k i v}^{\mathrm{S}}+\rho^{\mathrm{PO}} \beta_{i k i v}^{\mathrm{S}} \sum_{l=1, l \neq i}^{L} \beta_{l k i v}^{\mathrm{S}}}{1+\rho^{\mathrm{PO}} \sum_{l=1}^{L} \beta_{l k i v}^{\mathrm{S}}} .
\end{gathered}
$$

The above results show how the estimated channels of two conventional linear estimators are contaminated by the channels of UEs from other cells using the same training sequences. The pilot-only (PO) MMSE estimation is set as the benchmark and will be compared with the DA method.

\section{B. Uplink Data Transmission}

In this phase, all UEs send their UL data at the power of $P_{\mathrm{D}}$ and the UL data is modulated to combat the strong 
co-channel interference. For the sake of simplicity, BPSK modulation is adopted although higher-order modulations can also be utilized: 4QAM-modulated uplink data is considered in simulations as an example. Let $\mathbf{x}_{i k} \in \mathbb{C}^{1 \times \tau_{\mathrm{D}}}$ denote the UL data sequences of $\mathrm{UE}_{i k}$ whilst $\mathbf{X}_{i}=\left[\mathbf{x}_{i 1}^{\mathrm{H}} \ldots \mathbf{x}_{i K}^{\mathrm{H}}\right]^{\mathrm{H}}$ denote the UL data matrix of cell $i$. Each UL data symbol is randomly selected from the set $\left\{\sqrt{P_{\mathrm{D}}},-\sqrt{P_{\mathrm{D}}}\right\}$.

Thus, the received signals during the UL data transmission at the MBS and the $v$-th SBS in cell $i$ can be expressed as

$$
\begin{aligned}
& \mathbf{Y}_{i 0}^{\mathrm{UD}}=\sum_{l=1}^{L} \mathbf{H}_{l i} \mathbf{X}_{l}+\mathbf{N}_{i 0}^{\mathrm{UD}}, \\
& \mathbf{Y}_{i v}^{\mathrm{UD}}=\sum_{l=1}^{L} \mathbf{G}_{l i v} \mathbf{X}_{l}+\mathbf{N}_{i v}^{\mathrm{UD}},
\end{aligned}
$$

where $\mathbf{N}_{i 0}^{\mathrm{UD}}$ and $\mathbf{N}_{i v}^{\mathrm{UD}}$ are AWGN matrices.

By utilizing the estimated CSI and the MMSE data detector, and applying the decoder under maximum a posteriori probability (MAP) criterion, the recovered UL data vector of $\mathrm{UE}_{i k}$, which connects to the $v$-th SBS or the MBS in cell $i$, is given as [21]

$$
\begin{gathered}
\hat{\mathbf{x}}_{i k}=\operatorname{Decoder}\left[\mathbf{a}_{i k}\right], \\
\mathbf{a}_{i k}= \begin{cases}\hat{\mathbf{h}}_{i k i}^{\mathrm{H}}\left(\hat{\mathbf{H}}_{i i} \hat{\mathbf{H}}_{i i}^{\mathrm{H}}+\rho_{i k 0}^{-1} \mathbf{I}_{M}\right)^{-1} \mathbf{Y}_{i 0}^{\mathrm{UD}}, & \mathcal{U}_{i k}=0, \\
\hat{\mathbf{g}}_{i k i v}^{\mathrm{H}}\left(\hat{\mathbf{G}}_{i i v} \hat{\mathbf{G}}_{i i v}^{\mathrm{H}}+\rho_{i k v}^{-1} \mathbf{I}_{M}\right)^{-1} \mathbf{Y}_{i v}^{\mathrm{UD}}, & \mathcal{U}_{i k}=v,\end{cases}
\end{gathered}
$$

and

$$
\begin{aligned}
& \rho_{i k 0}=\left(\sum_{k=1}^{K} \tilde{\beta}_{i k i}^{\mathrm{M}}+\sum_{l=1, l \neq i}^{L} \sum_{k=1}^{K} \beta_{l k i}^{\mathrm{M}}+\frac{N_{0}}{P_{\mathrm{D}}}\right)^{-1}, \\
& \rho_{i k v}=\left(\sum_{k=1}^{K} \tilde{\beta}_{i k i v}^{\mathrm{S}}+\sum_{l=1, l \neq i}^{L} \sum_{k=1}^{K} \beta_{l k i v}^{\mathrm{S}}+\frac{N_{0}}{P_{\mathrm{D}}}\right)^{-1},
\end{aligned}
$$

where $\hat{\mathbf{h}}_{i k i}, \hat{\mathbf{H}}_{i i}, \hat{\mathrm{g}}_{i k i v}, \hat{\mathbf{G}}_{i i v}$ are the estimated channels in (10) (11) and $\tilde{\beta}_{i k i}^{\mathrm{M}}, \tilde{\beta}_{i k i v}^{\mathrm{S}}$ are shown by (12).

At the end of this stage, decoded data at each SBS will be transferred to its central MBS via wired fiber such that the issues of error and latency can be neglected for the ease of subsequent analysis.

\section{DA MMSE Channel Estimation}

DA MMSE channel estimation performed at each MBS utilizes the decoded UL data from SBSs and known training sequences to jointly reconstruct the channels of decoupled UEs. Thus, the received UL signal of the first two stages at the MBS in cell $i$ can be jointly written as

$$
\mathbf{Y}_{i 0}^{\mathrm{TD}}=\sum_{l=1}^{L} \mathbf{H}_{l i} \mathbf{W}_{l}+\mathbf{Z}_{i 0}^{\mathrm{TD}},
$$

where $\mathbf{W}_{l}=\left[\mathbf{S}, \mathbf{X}_{l}\right], \mathbf{Z}_{i 0}^{\mathrm{TD}}=\left[\mathbf{N}_{i 0}^{\mathrm{UT}}, \mathbf{N}_{i 0}^{\mathrm{UD}}\right]$ and “TD" stands for the combination of training and data stages. Thus, the DA MMSE channel estimator for the $k$-th decoupled UE in cell $i$ is formulated as [20]

$$
\begin{aligned}
\hat{\mathbf{h}}_{i k i}^{\mathrm{DA}} & =\mathbf{Y}_{i 0}^{\mathrm{TD} *} \mathbf{C}_{i k i}^{\mathrm{TD}}, \\
\mathbf{C}_{i k i}^{\mathrm{TD} *} & =\arg \min _{\mathbf{C}_{i k i}^{\mathrm{TD}}} \mathbb{E}\left[\left\|\hat{\mathbf{h}}_{i k i}^{\mathrm{DA}}-\mathbf{h}_{i k i}\right\|_{2}^{2}\right],
\end{aligned}
$$

where the optimal solution of $\mathbf{C}_{i k i}^{\mathrm{TD}}$ and its performance analysis will be given in the next section.

\section{Downlink Data Transmission}

After the above three stages, MBSs have acquired the channels of their serving UEs in DL by using the PO method for MUEs and the DA method for DeUEs. Let $\Phi_{i 0}^{\mathrm{M}}=$ $\left\{k \mid \mathcal{D}_{i k}=\mathcal{U}_{i k}=0\right\}, \Phi_{i 0}^{\mathrm{D}}=\left\{k \mid \mathcal{D}_{i k}=0, \mathcal{U}_{i k} \neq 0\right\}$ denote the index sets of MUEs and DeUEs served by the MBS in cell $i$ and the cardinality of sets $\Phi_{i 0}^{\mathrm{M}}, \Phi_{i 0}^{\mathrm{D}}$ are $K_{i 0}^{\mathrm{M}}, K_{i 0}^{\mathrm{D}}$, the total number of DL UEs is $K_{i 0}^{\mathrm{dl}}=K_{i 0}^{\mathrm{D}}+K_{i 0}^{\mathrm{M}}$. Thus, the aggregate estimated DL channels at the MBS can be expressed as

$$
\hat{\mathbf{H}}_{i}^{\mathrm{dl}}=\left[\left[\hat{\mathbf{h}}_{i k_{1} i}^{\mathrm{MMSE}}\right]_{k_{1} \in \Phi_{i 0}^{\mathrm{M}}},\left[\hat{\mathbf{h}}_{i k_{2} i}^{\mathrm{DA}}\right]_{k_{2} \in \Phi_{i 0}^{\mathrm{D}}}\right] \in \mathbb{C}^{M \times K_{i 0}^{\mathrm{dl}} .}
$$

Then, ZF precoding is performed to cancel the inter-user interference with a proper vector normalization. The DL precoding matrix at the MBS of cell $i$ is denoted as $\mathbf{V}_{i 0}$ with the $k$-th column $\mathbf{v}_{i 0 k}=\frac{\hat{\mathbf{v}}_{i 0 k}}{\left\|\hat{\mathbf{v}}_{i 0 k}\right\|_{2}}$ and $\hat{\mathbf{v}}_{i 0 k}$ being the $k$-th column of $\hat{\mathbf{V}}_{i 0}, \hat{\mathbf{V}}_{i 0}$ is given by

$$
\hat{\mathbf{V}}_{i 0}=\hat{\mathbf{H}}_{i}^{\mathrm{dl}}\left(\left(\hat{\mathbf{H}}_{i}^{\mathrm{dl}}\right)^{\mathrm{H}} \hat{\mathbf{H}}_{i}^{\mathrm{dl}}\right)^{-1} .
$$

Meanwhile, SBSs perform ZF IN precoding to serve their DL UEs and cancel the interference to DeUEs by utilizing the estimated UL channels of DeUEs at their UL SBSs. Let $\Phi_{i v}^{\mathrm{S}}=$ $\left\{k \mid \mathcal{D}_{i k}=\mathcal{U}_{i k}=v\right\}, \Phi_{i v}^{\mathrm{D}}=\left\{k \mid \mathcal{D}_{i k}=0, \mathcal{U}_{i k}=v\right\}$ denote the index sets of SUEs and DeUEs served by the $v$-th SBS of cell $i$ in UL and the cardinality of two sets is $K_{i v}^{\mathrm{S}}, K_{i v}^{\mathrm{D}}$, Note that the total number of DL UEs is defined as $K_{i v}^{\mathrm{dl}}=K_{i v}^{\mathrm{S}}$. The aggregate estimated channel matrices of SUEs and DeUEs are given as

$$
\begin{aligned}
& \hat{\mathbf{G}}_{i v}^{\mathrm{S}}=\left[\left[\hat{\mathbf{g}}_{i k i v}^{\mathrm{MMSE}}\right]_{k \in \Phi_{i v}^{\mathrm{S}}}\right] \in \mathbb{C}^{N \times K_{i v}^{\mathrm{S}}}, \\
& \hat{\mathbf{G}}_{i v}^{\mathrm{D}}=\left[\left[\hat{\mathbf{g}}_{i k i v}^{\mathrm{MMSE}}\right]_{k \in \Phi_{i v}^{\mathrm{D}}}\right] \in \mathbb{C}^{N \times K_{i v}^{\mathrm{D}} .}
\end{aligned}
$$

Then, the ZF IN precoding matrix at the $v$-th SBS of cell $i$ is denoted as $\mathbf{V}_{i v}$ with the $k$-th column $\mathbf{v}_{i v k}=\frac{\hat{\mathbf{v}}_{i v k} \|_{i}}{\left\|\hat{\mathbf{v}}_{i v k}\right\|_{2}}$ and $\hat{\mathbf{v}}_{i v k}$ being the $k$-th column of $\hat{\mathbf{V}}_{i v}$ defined as

$$
\hat{\mathbf{V}}_{i v}=\left(\mathbf{I}_{N}-\hat{\mathbf{U}}_{i v} \hat{\mathbf{U}}_{i v}^{\mathrm{H}}\right) \hat{\mathbf{G}}_{i v}^{\mathrm{S}}\left(\left(\hat{\mathbf{G}}_{i v}^{\mathrm{S}}\right)^{\mathrm{H}} \hat{\mathbf{G}}_{i v}^{\mathrm{S}}\right)^{-1},
$$

where $\hat{\mathbf{U}}_{i v}$ is formed by the eigenvectors of $\hat{\mathbf{G}}_{i v}^{\mathrm{D}}\left(\hat{\mathbf{G}}_{i v}^{\mathrm{D}}\right)^{\mathrm{H}}$ associated with its non-zero eigenvalues.

Hence, the received DL signal at $\mathrm{UE}_{i k}$ is expressed as $y_{i k}^{\mathrm{DL}}=\sum_{l=1}^{L}\left(\sqrt{\frac{P_{\mathrm{M}}}{K_{l 0}^{\mathrm{dl}}}} \mathbf{h}_{i k l}^{\mathrm{H}} \mathbf{V}_{l 0} \mathbf{c}_{l 0}+\sum_{s=1}^{S} \sqrt{\frac{P_{\mathrm{S}}}{K_{l s}^{\mathrm{dl}}}} \mathbf{g}_{i k l s}^{\mathrm{H}} \mathbf{V}_{l s} \mathbf{c}_{l s}\right)+z_{i k}^{\mathrm{DL}}$,

where $\mathbf{c}_{l s} \sim \mathcal{C N}\left(\mathbf{0}_{K_{l s}^{\mathrm{dl}}}, \mathbf{I}_{K_{l s}^{\mathrm{dl}}}\right)$ is the i.i.d. DL data vector and $z_{i k}^{\mathrm{DL}}$ is the AWGN. This is the end of a typical frame and more theoretical analysis will be given in the next section.

\section{ANALYSis AND Discussion}

In this section, we will give the specific formation of the DA MMSE channel estimator and analyze its NMSE performance, 
which will be compared with conventional linear estimators. Moreover, the achievable DL rate using the proposed transmission scheme will be derived and the effects of DA MMSE channel estimation and ZF IN precoding will also be discussed.

\section{A. DA MMSE Channel Estimator}

In order to evaluate the performance of different estimators, NMSE is adopted as performance metric and is defined as [22]

$$
\operatorname{NMSE}=10 \log _{10}\left(\frac{\mathbb{E}\left[\|\mathbf{h}-\hat{\mathbf{h}}\|_{2}^{2}\right]}{\mathbb{E}\left[\|\mathbf{h}\|_{2}^{2}\right]}\right) \quad(\text { in } \mathrm{dB}),
$$

where $\mathbf{h}$ is generically the true vector and $\hat{\mathbf{h}}$ is the estimated one.

To perform the DA MMSE channel estimator in Sec. III-C, the main challenge is how to utilize the decoded UL sequence with random errors we are unable to locate. Following the approach in [11], by introducing the error matrix $\mathbf{E}_{i}$ and the estimated aggregate UL matrix $\hat{\mathbf{W}}_{i}$, we can write the aggregate matrix of UL signal in cell $i$ as

$$
\mathbf{W}_{i}=\hat{\mathbf{W}}_{i} \circ \mathbf{E}_{i}=\left[\mathbf{S}, \hat{\mathbf{X}}_{i}\right] \circ\left[\mathbf{E}_{i}^{\mathrm{T}}, \mathbf{E}_{i}^{\mathrm{D}}\right],
$$

where $\hat{\mathbf{X}}_{i}=\left[\hat{\mathbf{x}}_{i 1}^{\mathrm{H}} \ldots \hat{\mathbf{x}}_{i K}^{\mathrm{H}}\right]^{\mathrm{H}}$ and $\circ$ is the Hadamard product; $\mathbf{E}_{i}^{\mathrm{T}}$ and $\mathbf{E}_{i}^{\mathrm{D}}$ are error matrices of UL training and data sequences, respectively. As training sequences are known at MBSs, $\mathbf{E}_{i}^{\mathrm{T}}$ is an all-one matrix. The element of $\mathbf{E}_{i}^{\mathrm{D}}$ is 1 or -1 where -1 means that the corresponding symbol is decoded incorrectly. With the assumptions that symbols are transmitted equally and decoded symbols in each UL data sequence have the same probability of error independently, the first two moments of $e_{i k j}=\left[\mathbf{E}_{i}^{\mathrm{D}}\right]_{k j}$ can be given as

$$
\begin{aligned}
\mathbb{E}\left[e_{i k j}\right] & =1-2 \mathrm{BER}_{i k v}, \\
\mathbb{E}\left[\left|e_{i k j}\right|^{2}\right] & =1,
\end{aligned}
$$

where $\mathrm{BER}_{i k v}$ is the BER value of the decoded UL sequence of $\mathrm{UE}_{i k}$ served by the $v$-th SBS.

As the expression of BER is intractable, Theorem 1 proposes a method to obtain an approximated analytical BER expression using moment matching and random matrix theory. Note that similar methods are also used in [19] and [23].

Theorem 1: Applying the proposed transmission scheme in a multi-cell HetNet with decoupling access, the UL data BER of the $k$-th UE served by the $v$-th SBS in UL of cell $i$ can be calculated approximately as

$$
\begin{aligned}
\operatorname{BER}_{i k v} & \approx \frac{\Gamma\left(\alpha_{i k v}+\frac{1}{2}\right)}{\Gamma\left(\alpha_{i k v}\right) 2 \sqrt{2 \pi}} \frac{\xi_{i k v}^{-\alpha_{v k}}}{\alpha_{i k v}\left(\frac{1}{\xi_{i k v}}+\frac{1}{2}\right)^{\alpha_{i k v}+\frac{1}{2}}} \\
& \times{ }_{2} F_{1}\left(1, \alpha_{i k v}+\frac{1}{2} ; \alpha_{i k v}+1 ; \frac{\frac{1}{\xi_{i k v}}}{\frac{1}{\xi_{i k v}}+\frac{1}{2}}\right),
\end{aligned}
$$

where $\Gamma(\cdot)$ is the gamma function and ${ }_{2} F_{1}(\cdot)$ is the hypergeometric function; $\alpha_{i k v}=N \frac{\mu_{i k v}^{2}}{\sigma_{i k v}^{2}}$ and $\xi_{i k v}=\rho_{i k v} \hat{\beta}_{i k i v}^{\mathrm{S}} \frac{\sigma_{i k v}^{2}}{\mu_{i k v}}$.

If $N$ and $K$ both converge to infinity while the ratio $c=$
$\frac{K-1}{N}$ converges to a positive quantity [24], ${ }^{2}$ and $\varpi_{i k v j}=$ $N \rho_{i k v} \hat{\beta}_{i j i v}^{\mathrm{S}}, \mu_{i k v}$ and $\sigma_{i k v}^{2}$ can be obtained by solving the following equations

$$
\begin{array}{r}
\mu_{i k v}=(1-c)+\frac{c}{K-1} \sum_{j=1}^{K-1} \frac{1}{\mu_{i k v} \varpi_{i k v j}+1}, \\
\sigma_{i k v}^{2}=1-c-\frac{c}{K-1} \sum_{j=1}^{K-1} \frac{\varpi_{i k v j} \sigma_{i k v}^{2}}{\left(\mu_{i k v} \varpi_{i k v j}+1\right)^{2}} \\
+\frac{c}{K-1} \sum_{j=1}^{K-1} \frac{1}{\mu_{i k v} \varpi_{i k v j}+1} .
\end{array}
$$

Proof: See Appendix A.

Remark 1: With the help of these analytical equations, we could estimate the BER values of UEs' UL data at BSs only with long-term parameters which are already known and change slowly. Moreover, once the calculation is finished at each SBS then the BER values are sent to the MBSs via backhaul links. Such a strategy not only offloads the computation to SBSs but reduces the feedback overhead.

Hence, DA MMSE estimator can be derived by utilizing the decoded UL data sequences and estimated BER values.

Theorem 2: Applying the proposed transmission scheme in a multi-cell HetNet with DUDe, the estimated channel between a DeUE $\mathrm{UE}_{i k}$ to the MBS in cell $i$ using the DA MMSE estimator is given as

$$
\begin{aligned}
\hat{\mathbf{h}}_{i k i}^{\mathrm{DA}} & =\mathbf{Y}_{i 0}^{\mathrm{TD}} \mathbf{C}_{i k i}^{\mathrm{TD}}, \\
\mathbf{C}_{i k i}^{\mathrm{TD}} & =\left(\hat{\mathbf{P}}+\Delta \mathbf{P}+N_{0} \mathbf{I}_{\left(\tau_{\mathrm{T}}+\tau_{\mathrm{D}}\right)}\right)^{-1}\left(\hat{\mathbf{w}}_{i k} \circ \overline{\mathbf{e}}_{i k}\right)^{\mathrm{H}} \beta_{i k i}^{\mathrm{M}}, \\
\hat{\mathbf{P}} & =\left(\left[\mathbf{S}, \hat{\mathbf{X}}_{i} \circ \overline{\mathbf{E}}_{i}^{\mathrm{D}}\right]\right)^{\mathrm{H}} \mathbf{R}_{\mathbf{H}_{i i}}\left(\left[\mathbf{S}, \hat{\mathbf{X}}_{i} \circ \overline{\mathbf{E}}_{i}^{\mathrm{D}}\right]\right), \\
\Delta \mathbf{P} & =\left[\begin{array}{c}
\mathbf{S}^{\mathrm{H}}\left(\sum_{l=1, l \neq i}^{L} \mathbf{R}_{\mathbf{H}_{l i}}\right) \mathbf{S} \\
\mathbf{0} \\
\left.\left(P_{\mathrm{D}}\left(\sum_{l=1, l \neq i}^{L} \sum_{k=1}^{K} \beta_{l k i}^{\mathrm{M}}\right)+S_{\mathbf{X}}\right) \mathbf{I}\right]
\end{array}\right] \\
S_{\mathbf{X}} & =P_{\mathrm{D}} \sum_{k=1}^{K} \beta_{i k i}^{\mathrm{M}}\left\{1-\left(1-2 \mathrm{BER}_{i k v}\right)^{2}\right\},
\end{aligned}
$$

where $\hat{\mathbf{w}}_{i k}, \overline{\mathbf{e}}_{i k}$ are the $k$-th column of $\hat{\mathbf{W}}_{l}, \overline{\mathbf{E}}_{i}$, and $\overline{\mathbf{E}}_{i}=$ $\mathbb{E}\left[\mathbf{E}_{i}\right]$, respectively.

The NMSE of the estimated channel from a DeUE $\mathrm{UE}_{i k}$ to the MBS in cell $i$ using the DA MMSE estimator can be calculated asymptotically as

$$
\mathcal{J}_{i k}^{\mathrm{DA}, \mathrm{PC}} \simeq 10 \log _{10}\left(\frac{1}{1+\rho_{i k}^{\mathrm{DA}, \mathrm{PC}} \beta_{i k i}^{\mathrm{M}}}\right)
$$

where $\simeq$ means "asymptotically equal to" and

$$
\begin{aligned}
\rho_{i k}^{\mathrm{DA}, \mathrm{PC}} & =\frac{\rho^{\mathrm{PO}}}{1+\rho^{\mathrm{PO}} \sum_{l=1, l \neq i}^{L} \beta_{l k i}^{\mathrm{M}}}+\frac{\tau_{\mathrm{D}} P_{\mathrm{D}}\left(1-2 \mathrm{BER}_{i k v}\right)^{2}}{p_{\mathrm{A}, 22}}, \\
p_{\mathrm{A}, 22} & =\left(P_{\mathrm{D}}\left(\sum_{l=1, l \neq i}^{L} \sum_{k=1}^{K} \beta_{l k i}^{\mathrm{M}}\right)+S_{\mathbf{X}}+N_{0}\right) .
\end{aligned}
$$

${ }^{2}$ If $N$ and $K$ is not very large, these equations are good approximations [23] and still works when $N<K$ [11]. 
Proof: See Appendix B.

The NMSE performances of classic PO and DA channel estimators in a single-cell or a multi-cell HetNet are summarized in Table I with some insights concluded below.

Remark 2: The above results show that SNR-like parameter $\rho^{\mathrm{DA}}$ for DA MMSE estimators equals $\rho^{\mathrm{PO}}$ for PO estimators plus an offset term. Although this offset has different forms in single-cell and multi-cell scenarios, we have $\rho^{\mathrm{DA}} \geq \rho^{\mathrm{PO}}$, and thus it can be inferred that DA methods always have better NMSE performance than conventional PO methods. Moreover, the NMSE of the DA method in multi-cell can be regarded as the general NMSE expression and, by setting $P_{\mathrm{D}}=0$ ( or $\tau_{\mathrm{D}}=0$ ) and $L=1$, (38) reduces to the NMSE of using the PO method and the single-cell scenario, respectively.

Remark 3: As can be seen from (38)-(40) increasing $\tau_{\mathrm{D}}$ leads to higher $\rho$ and by letting $\tau_{\mathrm{D}} \rightarrow \infty$, we have the perfect CSI case. However, when $P_{\mathrm{D}}$ is increased continuously, $\rho_{i k}^{\mathrm{DA}, \mathrm{PC}}$ in (40) is upper bounded by

$$
\dot{\rho}_{i k}^{\mathrm{DA}, \mathrm{PC}}=\rho_{i k}^{\mathrm{PO}, \mathrm{PC}}+\frac{\tau_{\mathrm{D}}\left(1-2 \mathrm{BER}_{i k v}\right)^{2}}{\sum_{l=1, l \neq i}^{L} \sum_{k=1}^{K} \beta_{l k i}^{\mathrm{M}}+\sum_{k=1}^{K} \beta_{i k i}^{\mathrm{M}}\left\{1-\left(1-2 \mathrm{BER}_{i k v}\right)^{2}\right\}},
$$

which means that increasing the data power infinitely cannot bring sustainable benefit to $\rho$ since the interference in the DA method is also increased. In addition, improving BER is another way to gain better $\rho$ and when BER values approaching to $0, \rho_{i k}^{\mathrm{DA}, \mathrm{PC}}$ is upper bounded by

$$
\ddot{\rho}_{i k}^{\mathrm{DA}, \mathrm{PC}}=\rho_{i k}^{\mathrm{PO}, \mathrm{PC}}+\frac{\tau_{\mathrm{D}} P_{\mathrm{D}}}{P_{\mathrm{D}} \sum_{l=1, l \neq i}^{L} \sum_{k=1}^{K} \beta_{l k i}^{\mathrm{M}}+N_{0}},
$$

where the effect of intra-cell interference is completely eliminated.

\section{B. Achievable Downlink Rate}

Next, we will analyze the achievable DL rate of DeUEs using the proposed transmission scheme. First, we introduce a lemma required to obtain the closed-form expression of the achievable DL rate.

Lemma 1: By using the DA MMSE estimator at the MBS of cell $i$, the estimated channel of $\mathrm{UE}_{i k}$ can be approximated as (43) and its error vector is defined as

$$
\tilde{\mathbf{h}}_{i k i}^{\mathrm{DA}}=\mathbf{h}_{i k i}-\hat{\mathbf{h}}_{i k i}^{\mathrm{DA}},
$$

where $\chi_{i k}=\hat{\mathbf{x}}_{i k} \circ \mathbf{e}_{i k}^{\mathrm{D}}$ and $\bar{\chi}_{i k}=\hat{\mathbf{x}}_{i k} \circ \overline{\mathbf{e}}_{i k}^{\mathrm{D}}$. Also, the two vectors are distributed as

$$
\hat{\mathbf{h}}_{i k i}^{\mathrm{DA}} \sim \mathcal{C N}\left(0, \hat{\beta}_{i k i}^{\mathrm{M}, \mathrm{DA}} \mathbf{I}_{M}\right), \tilde{\mathbf{h}}_{i k i}^{\mathrm{DA}} \sim \mathcal{C N}\left(0, \tilde{\beta}_{i k i}^{\mathrm{M}, \mathrm{DA}} \mathbf{I}_{M}\right)
$$

where

$$
\hat{\beta}_{i k i}^{\mathrm{M}, \mathrm{DA}}=\frac{\left(\beta_{i k i}^{\mathrm{M}}\right)^{2} \rho_{k}^{\mathrm{DA}, \mathrm{PC}}}{1+\beta_{i k i}^{\mathrm{M}} \rho_{k}^{\mathrm{DA}, \mathrm{PC}}}, \quad \tilde{\beta}_{i k i}^{\mathrm{M}, \mathrm{DA}}=\frac{\beta_{i k i}^{\mathrm{M}}}{1+\beta_{i k i}^{\mathrm{M}} \rho_{k}^{\mathrm{DA}, \mathrm{PC}}} .
$$

Proof: See Appendix C.

Remark 4: The above lemma shows that the channels estimated by DA MMSE estimator contain not only the channels using the same pilot but channels using orthogonal pilots which results from interference during UL data transmission. As the coefficient $\chi_{l j} \bar{\chi}_{i k}^{\mathrm{H}}$ is relatively small when $l \neq i$ or $j \neq k$, the effect of channels using orthogonal pilots is insignificant and the correlation between them is negligible. Moreover, we observe that $\beta_{i k i}=\hat{\beta}_{i k i}^{\mathrm{M}, \mathrm{DA}}+\tilde{\beta}_{i k i}^{\mathrm{M}, \mathrm{DA}}$, which implies that $\hat{\mathbf{h}}_{i k i}^{\mathrm{DA}}$ and $\tilde{\mathbf{h}}_{i k i}^{\mathrm{DA}}$ are asymptotical uncorrelated when $\hat{\mathbf{X}} \hat{\mathbf{X}}^{\mathrm{H}} \rightarrow \tau_{\mathrm{D}} P_{\mathrm{D}} \mathbf{I}_{\tau_{\mathrm{D}}}$.

Next, we utilize the achievable rate analysis developed in [27] and the received DL signal at $\mathrm{UE}_{i k}$ in (26) that can be expanded as in (47) where $\mathbb{E}\left[\mathbf{h}_{i k i}^{\mathrm{H}} \mathbf{v}_{i 0[k]}\right]$ is the effective channel known at $\mathrm{UE}_{i k}$ and $\sum_{j=1}^{7} I_{i k}^{(j)}$ is the effective noise. Specifically, $S_{i k}$ is the desired signal, $I_{i k}^{(1)}$ is due to the difference between effective and instantaneous channels, $I_{i k}^{(2)}$ results from channel estimation error in cell $i, I_{i k}^{(3)}$ is interference from MBSs in other cells, $I_{i k}^{(4)}, I_{i k}^{(5)}, I_{i k}^{(6)}$ is interference from the SBS serving $\mathrm{UE}_{i k}$ in UL, other SBSs in cell $i$ and SBSs in other cells respectively. Note that the subscript $\left[k_{i}\right]$ of $\mathbf{v}_{i 0\left[k_{i}\right]}$ represents the index of $\mathrm{UE}_{i k}$ among DL UEs at the MBS in cell $i$. Then, we derive the following achievable rate by treating the additive noise as independent Gaussian noise, which can be regarded as the worst case. For simplicity, we assume that BSs outside the typical cell (cell $i$ ) perform MMSE channel estimation and the bandwidth is unit.

Theorem 3: Applying the proposed transmission scheme in a multi-cell decoupled HetNet, the achievable rate of $\mathrm{UE}_{i k}$ is calculated as

$$
\mathcal{R}_{i k}=\log _{2}\left(1+\theta_{i k}\right) \quad(\text { bits/sec }),
$$

where $\theta_{i k}$ is shown in (48) with $V_{i 0}=M-K_{i 0}^{\mathrm{dl}}+1, \Upsilon(p, q)=$ $\frac{\Gamma(q+p)}{\Gamma(q)}$ and

$$
\mathbb{E}\left[\left|I_{i k, l}^{(3)}\right|^{2}\right]= \begin{cases}\frac{\left(M-K_{l 0}^{\mathrm{dl}}+1\right)}{K_{l 0}^{\mathrm{dl}}} P_{\mathrm{M}} \hat{\beta}_{i k l}^{\mathrm{M}}+P_{\mathrm{M}} \tilde{\beta}_{i k l}^{\mathrm{M}} & \mathcal{D}_{l k}=0 \\ P_{\mathrm{M}} \beta_{i k l}^{\mathrm{M}} & \mathcal{D}_{l k} \neq 0\end{cases}
$$

$\mathbb{E}\left[\left|I_{i k, l s}^{(6)}\right|^{2}\right]=\left\{\begin{array}{lr}\xi_{l s} P_{\mathrm{S}}\left(\frac{\left(N-K_{l s}^{\mathrm{dl}}+1\right)}{K_{l s}^{\mathrm{dl}}} \hat{\beta}_{i k l s}^{\mathrm{S}}+\tilde{\beta}_{i k l s}^{\mathrm{S}}\right) & \mathcal{D}_{l k}=\mathcal{U}_{l k}=s, \\ \xi_{l s} P_{\mathrm{S}} \tilde{\beta}_{i k l s}^{\mathrm{S}} & \mathcal{U}_{l k}=s, \mathcal{D}_{l k} \neq s, \\ \xi_{l s} P_{\mathrm{S}} \beta_{i k l s}^{\mathrm{S}} & \mathcal{U}_{l k} \neq s, \mathcal{D}_{l k} \neq s .\end{array}\right.$

where $s=1, \ldots, S$. The indicator $\xi_{i v}$ equals 1 when the $v$ th SBS in cell $i$ is active in DL and equals 0 otherwise.

Proof: See Appendix D.

Remark 5: Compared with the conventional transmission scheme, DA MMSE estimator provides more reliable CSI with higher $\hat{\beta}_{i k i}^{\mathrm{M}, \mathrm{DA}}$ (lower $\tilde{\beta}_{i k i}^{\mathrm{M}}$ ) as shown in (39) and (46), thus higher SINR can be achieved in (48). Moreover, ZF IN precoding performed at DeUEs' UL SBSs almost cancels the strongest inter-tier interference to DeUEs and obtains better DL rate by reducing $\hat{\beta}_{i k i v}^{S}$ to $\tilde{\beta}_{i k i v}^{S}$ in (48).

Remark 6: When increasing $P_{\mathrm{D}}$ and $\tau_{\mathrm{D}}$ respectively for better DL rate, $\hat{\beta}_{i k i}^{\mathrm{M}, \mathrm{DA}}$ will be upper bounded by $\dot{\hat{\beta}}_{i k i}^{\mathrm{M}, \mathrm{DA}}=$ $\left.\hat{\beta}_{i k i}^{\mathrm{M}, \mathrm{DA}}\right|_{\rho_{i k}^{\mathrm{DA}, \mathrm{PC}}=\dot{\rho}_{i k}^{\mathrm{DA}, \mathrm{PC}}}$ and $\ddot{\hat{\beta}}_{i k i}^{\mathrm{M}, \mathrm{DA}}=\left.\hat{\beta}_{i k i}^{\mathrm{M}, \mathrm{DA}}\right|_{\rho_{i k}^{\mathrm{DA}, \mathrm{PC}} \rightarrow \infty}=\beta_{i k i}^{\mathrm{M}, \mathrm{DA}}$, thus the achievable DL rate is also bounded by

$$
\begin{aligned}
& \dot{\mathcal{R}}_{i k}=\left.\mathcal{R}_{i k}\right|_{\rho_{i k}^{\mathrm{DA}, \mathrm{PC}}}=\dot{\rho}_{i k}^{\mathrm{DA}, \mathrm{PC}}, \\
& \ddot{\mathcal{R}}_{i k}=\left.\mathcal{R}_{i k}\right|_{\rho_{i k}^{\mathrm{DA}, \mathrm{PC}} \rightarrow \infty},
\end{aligned}
$$

which can be also observed in our simulations. 
TABLE I

NMSE FOR DIFFERENT CHANNEL ESTIMATION METHODS

\begin{tabular}{|c|c|c|c|}
\hline & \multicolumn{2}{|c|}{ Conventional } & Data-Aided \\
\hline & $\mathbf{L S}$ & MMSE & MMSE \\
\hline \multirow[b]{2}{*}{ Single-Cell } & $10 \log _{10}\left(\frac{1}{\rho^{\mathrm{PO}} \beta_{k}^{\mathrm{M}}}\right)[16]$ & $10 \log _{10}\left(\frac{1}{1+\rho^{\mathrm{PO}} \beta_{k}^{\mathrm{M}}}\right)[25]$ & $10 \log _{10}\left(\frac{1}{1+\rho_{k}^{\mathrm{DA}} \beta_{k}^{\mathrm{M}}}\right)[11]$ \\
\hline & \multicolumn{2}{|c|}{$\rho^{\mathrm{PO}}=\frac{\tau_{\mathrm{T}} P_{\mathrm{T}}}{N_{0}}$} & $\begin{array}{c}\rho_{k}^{\mathrm{DA}}=\frac{\tau_{\mathrm{T}} P_{\mathrm{T}}}{N_{0}}+\frac{\tau_{\mathrm{D}} P_{\mathrm{D}}\left(1-2 \mathrm{BER}_{v k}\right)^{2}}{S_{\mathbf{X}}+N_{0}} \\
S_{\mathbf{X}}=P_{\mathrm{D}} \sum_{k}^{K} \beta_{k}^{\mathrm{M}}\left\{1-\left(1-2 \mathrm{BER}_{v k}\right)^{2}\right\}\end{array}$ \\
\hline \multirow[b]{2}{*}{$\begin{array}{l}\text { Multi-Cell } \\
\text { with PC }\end{array}$} & $10 \log _{10}\left(\frac{1}{\rho_{i k}^{\mathrm{PO} P C} \beta_{i k i}^{\mathrm{M}}}\right)$ & $10 \log _{10}\left(\frac{1}{1+\rho_{i k}^{\mathrm{PO}, \mathrm{PC}} \beta_{i k i}^{\mathrm{M}}}\right)[26]$ & $10 \log _{10}\left(\frac{1}{1+\rho_{i k}^{\mathrm{DA}, \mathrm{PC}} \beta_{i k i}^{\mathrm{M}}}\right)$ \\
\hline & \multicolumn{2}{|c|}{$\rho_{i k}^{\mathrm{PO}, \mathrm{PC}}=\frac{\rho^{\mathrm{PO}}}{1+\left(\sum_{l=1, l \neq i}^{L} \beta_{l k i}^{\mathrm{M}}\right) \rho^{\mathrm{PO}}}$} & $\begin{array}{c}\rho_{i k}^{\mathrm{DA}, \mathrm{PC}}=\rho_{i k}^{\mathrm{PO}, \mathrm{PC}}+\frac{\tau_{\mathrm{D}} P_{\mathrm{D}}\left(1-2 \mathrm{BER}_{i k v}\right)^{2}}{p_{\mathrm{A}, 22}} \\
p_{\mathrm{A}, 22}=P_{\mathrm{D}}\left(\sum_{l=1, l \neq i}^{L} \sum_{k=1}^{K} \beta_{l k i}^{\mathrm{M}}\right)^{2}+S_{\mathbf{X}}+N_{0} \\
S_{\mathbf{X}}=P_{\mathrm{D}} \sum_{k=1}^{K} \beta_{i k i}^{\mathrm{M}}\left\{1-\left(1-2 \mathrm{BER}_{i k v}\right)^{2}\right\}\end{array}$ \\
\hline
\end{tabular}

$$
\begin{aligned}
& \hat{\mathbf{h}}_{i k i}^{\mathrm{DA}} \approx \frac{\beta_{i k i}^{\mathrm{M}}}{1+\beta_{i k i}^{\mathrm{M}} \rho_{k}^{\mathrm{DA}, \mathrm{PC}}}\left\{\left(\rho_{i k}^{\mathrm{PO}, \mathrm{PC}}+\frac{\chi_{i k} \overline{\boldsymbol{\chi}}_{i k}^{\mathrm{H}}}{p_{\mathrm{A}, 22}}\right) \mathbf{h}_{i k i}+\sum_{j \neq k} \frac{\chi_{i j} \bar{\chi}_{i k}^{\mathrm{H}}}{p_{\mathrm{A}, 22}} \mathbf{h}_{i j i}+\sum_{l=1, l \neq i}^{L}\left(\rho_{i k}^{\mathrm{PO}, \mathrm{PC}} \mathbf{h}_{l k i}+\mathbf{H}_{l i} \mathbf{X}_{l} \frac{\overline{\boldsymbol{\chi}}_{i k}^{\mathrm{H}}}{p_{\mathrm{A}, 22}}\right)+\frac{\rho_{i k}^{\mathrm{PO}, \mathrm{PC}}}{\tau_{\mathrm{T}} P_{\mathrm{T}}} \mathbf{N}_{i 0}^{\mathrm{UT}} \mathbf{s}_{k}^{\mathrm{H}}+\frac{\mathbf{N}_{i 0}^{\mathrm{UD}} \bar{\chi}_{i k}^{\mathrm{H}}}{p_{\mathrm{A}, 22}}\right\}
\end{aligned}
$$

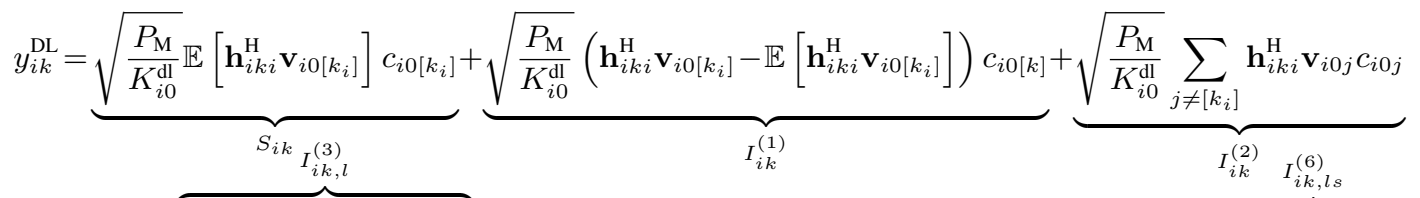

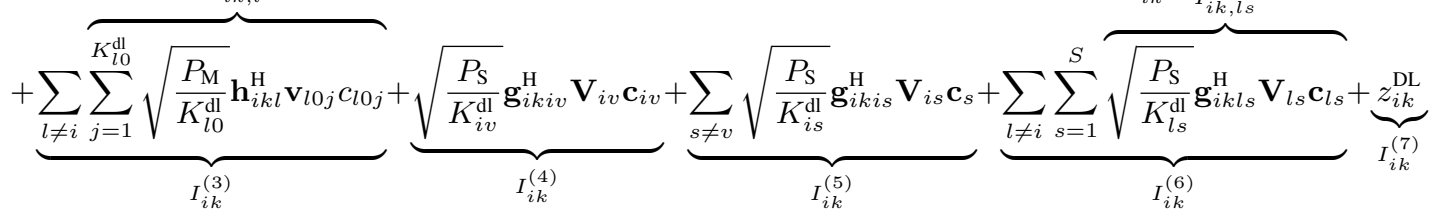

$$
\begin{aligned}
& \theta_{i k}=\frac{\frac{P_{\mathrm{M}} \hat{\beta}_{k i}^{\mathrm{M}, \mathrm{DA}}}{K_{i 0}^{\mathrm{d}}}}{\frac{P_{\mathrm{M}} \hat{\beta}_{i k i}^{\mathrm{M}, \mathrm{DA}}}{K_{i 0}^{\mathrm{dl}}}\left\{V_{i 0}-\Upsilon^{2}\left(\frac{1}{2}, V_{i 0}\right)\right\}+P_{\mathrm{M}} \tilde{\beta}_{i k i}^{\mathrm{M}, \mathrm{DA}}+\sum_{l \neq i} \mathbb{E}\left[\left|I_{i k, l}^{(3)}\right|^{2}\right]+\xi_{i v} P_{\mathrm{S}} \tilde{\beta}_{i k i v}^{\mathrm{S}}+\sum_{s \neq v} \xi_{i s} P_{\mathrm{S}} \beta_{i k i s}^{\mathrm{S}}+\sum_{l \neq i} \sum_{s=1}^{S} \mathbb{E}\left[\left|I_{i k, l s}^{(6)}\right|^{2}\right]+N_{0}}
\end{aligned}
$$

\section{NUMERICAL RESUlTS}

To validate the performance of the proposed scheme, the well-known 7-cell structure is adopted for simulations where the radius of each cell is 1000 meters. The central cell is termed as "typical cell" and to avoid edge effect we focus on the numerical results of the typical cell. Large-scale fading coefficients are defined as $\beta_{i k j}^{\mathrm{M}}=\left(d_{i k j}^{\mathrm{M}}\right)^{-\alpha}, \beta_{i k j v}^{\mathrm{S}}=\left(d_{i k j v}^{\mathrm{S}}\right)^{-\alpha}$ where $d_{i k j}^{\mathrm{M}}, d_{i k j v}^{\mathrm{S}}$ denote the distances between nodes and $\alpha$ is the attenuation exponent. Unless specified otherwise, the parameters in simulations are listed in Table II. We adopt two metrics to evaluate performance of the proposed scheme, one is the NMSE defined in (27) and the other is the average achievable DL rate defined as

$$
\mathcal{R}^{\mathrm{UE}}=\frac{1}{\operatorname{card}\left(\Phi^{\mathrm{UE}}\right)} \sum_{k \in \Phi^{\mathrm{UE}}} \mathcal{R}_{i k},
$$

where $\operatorname{card}(A)$ is the cardinality of set $A$ and cell $i$ is the typical cell. UE $\in\{$ MUE, SUE, DeUE $\}$ and $\Phi^{\mathrm{MUE}}=\Phi_{i 0}^{\mathrm{M}}$, $\Phi^{\text {DeUE }}=\Phi_{i 0}^{\mathrm{D}}, \Phi^{\mathrm{SUE}}=\bigcup_{v=1}^{S} \Phi_{i v}^{\mathrm{S}}$. The DL rate of a DeUE is calculated by (48) and (49) while the DL rates of a MUE and a SUE can be derived similarly by using the PO estimated
TABLE II

SIMULATION PARAMETERS

\begin{tabular}{c||c}
\hline \multicolumn{1}{c||}{ Parameter } & Value \\
\hline Number of cells $L$ & 7 \\
Number of SBSs per cell $S$ & 30 \\
Number of UEs per cell $K$ & 30 \\
Noise power spectral density $N_{0}$ & $-174 \mathrm{dBm} / \mathrm{Hz}$ \\
Attenuation exponent $\alpha$ & 4 \\
Antenna number of MBS $M$ & 256 \\
Antenna number of SBS $N$ & 8 \\
Transmit power of MBS $P_{\mathrm{M}}$ & $46 \mathrm{dBm}$ \\
Transmit power of SBS $P_{\mathrm{S}}$ & $24 \mathrm{dBm}$ \\
Training power of UEs $P_{\mathrm{T}}$ & $-7 \sim 23 \mathrm{dBm}$ \\
Data power of UEs $P_{\mathrm{D}}$ & $-7 \sim 23 \mathrm{dBm}$ \\
Training length $\tau_{\mathrm{T}}$ & 30 \\
Data length $\tau_{\mathrm{D}}$ & 128 \\
\hline
\end{tabular}

channels in (10) and (11).

Fig. 3 shows the BER performance of UL data sequences of DeUEs with different data detectors and perfect or imperfect CSI. As anticipated, we can observe that the MMSE detector with perfect CSI has the best performance, however, the gain 


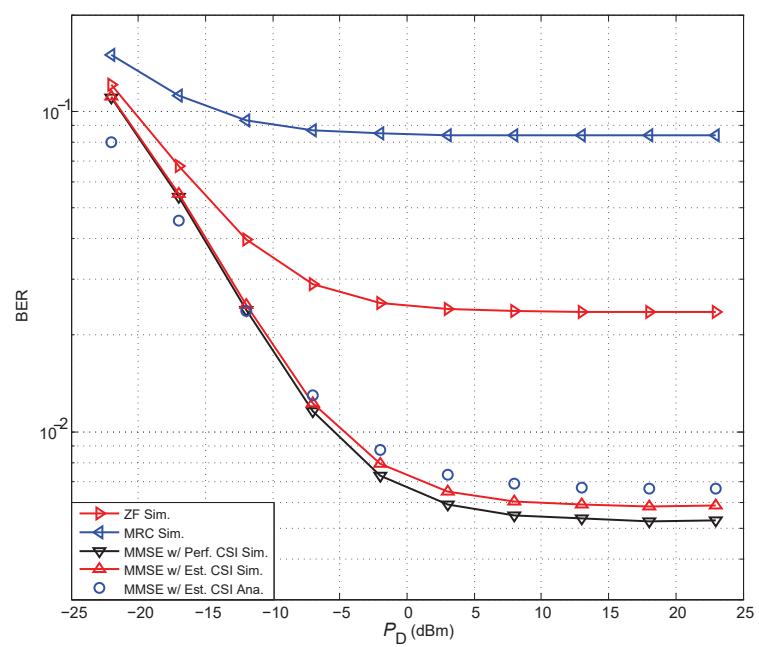

Fig. 3. UL data BER of DeUEs versus $P_{\mathrm{D}}$ with $P_{\mathrm{T}}=13 \mathrm{dBm}$.

over MMSE detector with estimated CSI is small and when increasing $P_{\mathrm{D}}$, the saturated BER is little better than $10^{-2}$ while the value is $10^{-3}$ for the single-cell scenario in [11]. This is due to the effect of pilot contamination and intercell interference. Besides, the analytical BER estimated by (31) almost matches the simulated BER with imperfect CSI, which verifies the accuracy of our calculations with long-term parameters in Theorem 1.

Fig. 4 and Fig. 5 plot the NMSE performance of the estimated DL channels of DeUEs with conventional PO and DA methods. The curves of conventional PO estimators serve as benchmarks and remain constant as $P_{\mathrm{D}}$ and $\tau_{\mathrm{D}}$ vary. In Fig. 4, note that when increasing $P_{\mathrm{D}}$, the curves of the DA methods first go down and then saturate to certain values gradually, which verifies the inference that there shall be a performance upper bound by increasing $P_{\mathrm{D}}$ in Remark 4. Despite this, DA methods can improve the NMSE performance of DeUEs by at least $8 \mathrm{~dB}$ when $P_{\mathrm{D}}$ is relatively large. Moreover, the NMSE for DA method without BER cannot decline log-linearly as the NMSE behaves as in the case of single-cell [11]. This is because when $P_{\mathrm{D}}$ grows, the SNR-like parameter $\rho$ without BER in (42) is upper bounded by $\rho_{i k}^{\mathrm{PO}, \mathrm{PC}}+\tau_{\mathrm{D}}\left(\sum_{l=1, l \neq i}^{L} \sum_{k=1}^{K} \beta_{l k i}^{\mathrm{M}}\right)^{-1}$, while (42) reduces to $\rho_{i k}^{\mathrm{PO}}+\frac{\tau_{\mathrm{D}} P_{\mathrm{D}}}{N_{0}}$ in single-cell scenario and $\rho$ can continuously increase. Thus, the downside of improving NMSE by increasing $P_{\mathrm{D}}$ is the inter-cell interference during the UL data stage. Besides, both numerical and analytical results show the NMSE without BER is only little better than the case with BER (at most $0.4 \mathrm{~dB}$ ), which indicates that the NMSE performance of DA methods is not sensitive to BER performance when BER is around $10^{-2}$, and there is a gap between analytical and simulated NMSE due to the approximation used in deriving the NMSE expression in (38).

Similar results are demonstrated in Fig. 5 except that NMSE for DA methods with or without BER can be reduced loglinearly by increasing $\tau_{\mathrm{D}}$, which is explained by (41) and (42). As expected, the simulated NMSE with or without BER converge to the analytical ones as $\tau_{\mathrm{D}}$ gets larger since the approximation is more accurate, while the NMSE without BER converges quicker. In addition, the NMSE performance

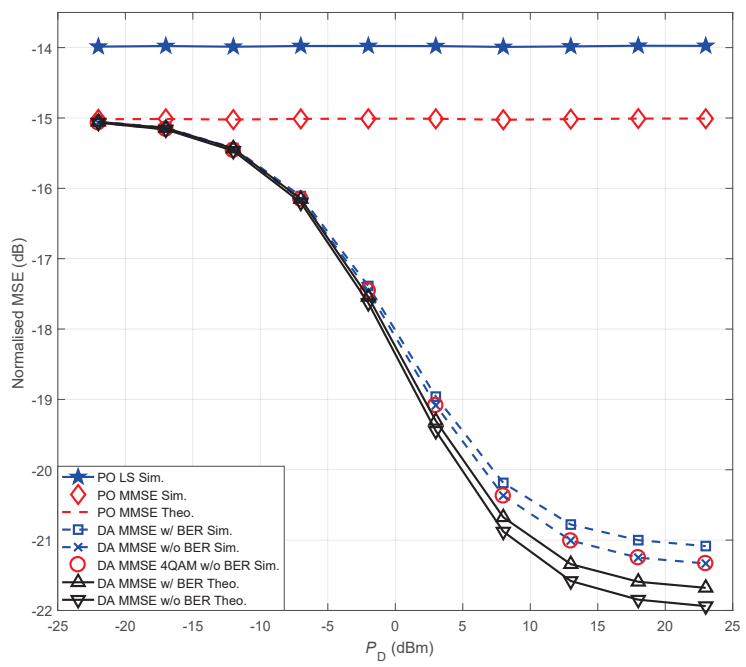

Fig. 4. NMSE of DeUEs versus $P_{\mathrm{D}}$ for different channel estimation methods with $P_{\mathrm{T}}=13 \mathrm{dBm}$.

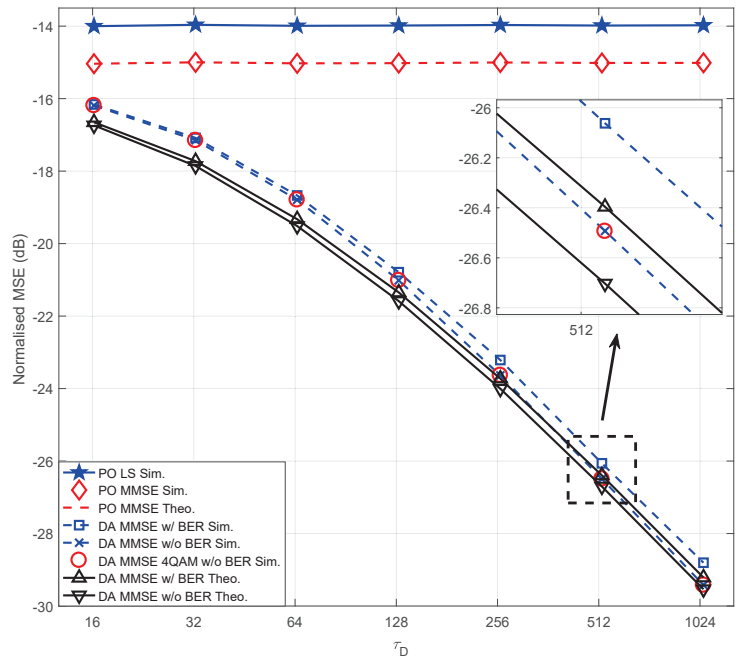

Fig. 5. NMSE of DeUEs versus $\tau_{\mathrm{D}}$ for different channel estimation methods with $P_{\mathrm{D}}=13 \mathrm{dBm}$ and $P_{\mathrm{T}}=13 \mathrm{dBm}$.

of 4 QAM without BER is also plotted and coincides with the NMSE for BPSK. Although the inference in [11], that the NMSE of the DA methods is determined by the total energy of training and UL data stages in the error-free scenario, is no longer valid in multi-cell, higher modulations can still achieve the same NMSE performance as BPSK does provided with the same training power and average UL data power.

Next, we examine the performance of the average achievable DL rate. Fig. 6 and Fig. 7 compare the average DL rates of MUEs, SUEs and DeUEs by using three different methods, namely PO, DA and DA plus IN. In both figures, we observe that the curves using PO method and curves of SUEs remain constant as expected. Rates of DeUEs can be improved firstly by using the DA method when increasing $P_{\mathrm{D}}$ or $\tau_{\mathrm{D}}$ and eventually approach the upper bounds derived in (50) and (51). The unexpected by-product of the DA method is that rates of MUEs can also be improved by at most $0.7 \mathrm{bps}$, which is because more accurate channels of DeUEs are utilized for DL precoding at MBSs. Then, combining DA with ZF IN precoding, the rates of DeUEs are further promoted by roughly $0.5 \mathrm{bps}$ as the result of nulling the nearest SBS interference 


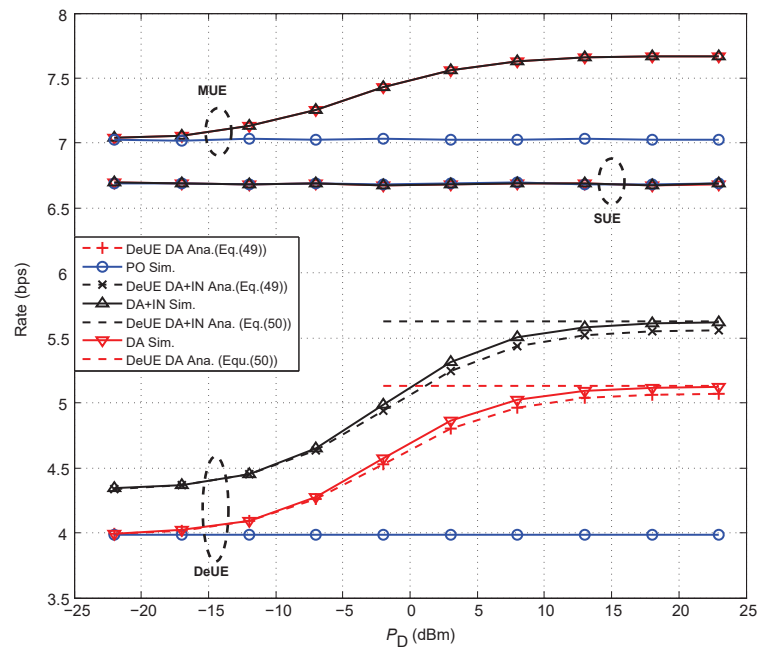

Fig. 6. Average achievable DL rates of MUEs, SUEs and DeUEs versus $P_{\mathrm{D}}$ with $P_{\mathrm{T}}=3 \mathrm{dBm}$.

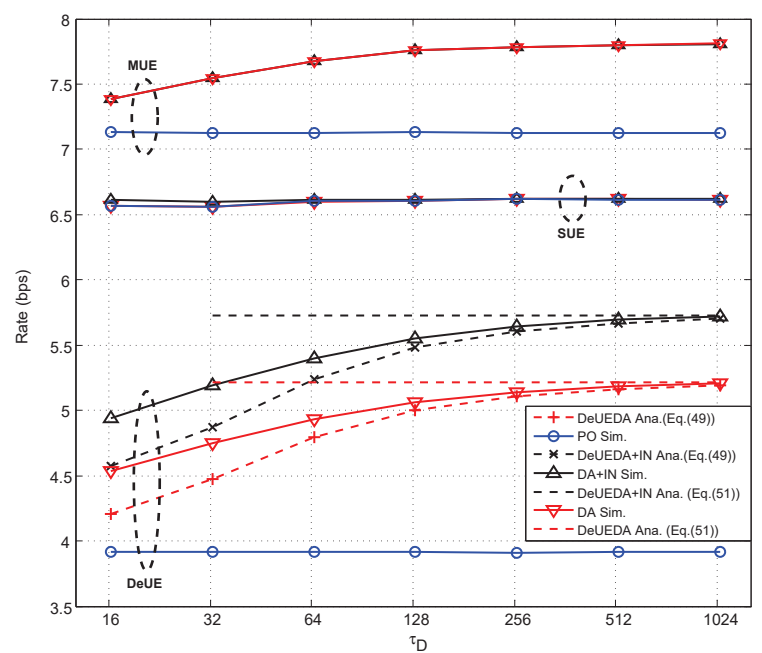

Fig. 7. Average achievable DL rates of MUEs, SUEs and DeUEs versus $\tau_{\mathrm{D}}$ with $P_{\mathrm{D}}=13 \mathrm{dBm}$ and $P_{\mathrm{T}}=3 \mathrm{dBm}$.

and noting that the rate gains from IN are nearly independent with $P_{\mathrm{D}}$ and $\tau_{\mathrm{D}}$. Unlike DA, IN precoding cannot bring extra benefit to MUEs. Moreover, the analytical results of DeUEs represented by dashed lines almost match the simulated ones in Fig. 6, while the gaps between two cases become larger when $\tau_{\mathrm{D}}$ gets smaller; this is because the approximation used in Lemma 3 is more accurate when UL data length is larger. Therefore, the DL performance of DeUEs can be enhanced by at most $1.8 \mathrm{bps}$ in total using the proposed transmission scheme and better DL rate fairness between three types of UEs is also achieved.

Fig. 8 and Fig. 9 compare the DL rates of three types of UEs in multi-cell and single-cell systems. As we can observe in Fig. 8 at $P_{\mathrm{D}}=23 \mathrm{dBm}$, compared with the single-cell scenario, the rates of all kinds of UEs using the PO method are decreased by about 0.5 bps while the rate gains with DA decrease from $1.8 \mathrm{bps}$ to $1.1 \mathrm{bps}$ for DeUEs and from $0.9 \mathrm{bps}$ to $0.6 \mathrm{bps}$ for MUEs in a multi-cell system. Also, the benefit of ZF IN precoding to DeUEs drops from 0.8 bps to $0.5 \mathrm{bps}$. Similar results can be observed in Fig. 9 when $\tau_{\mathrm{D}}$ varies. In view of two figures, we observe that with nearly perfect

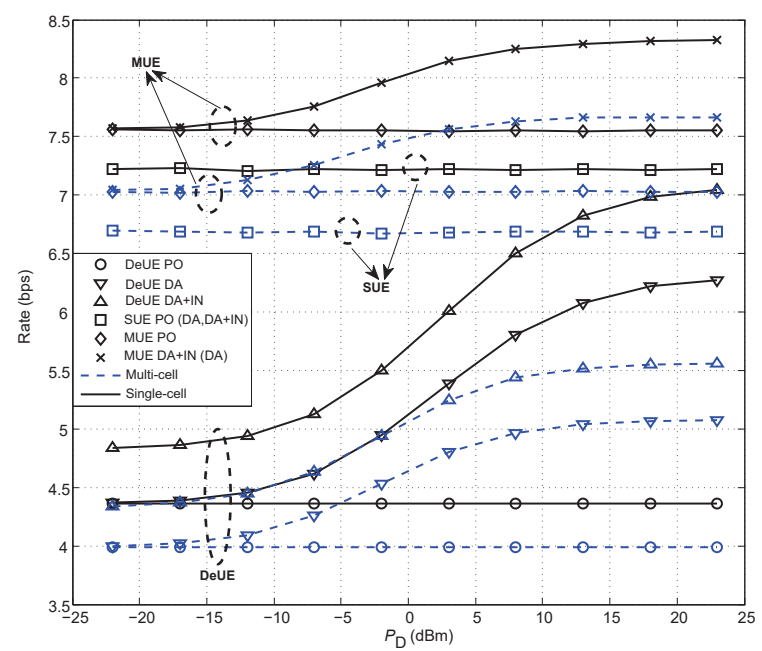

Fig. 8. Comparison of average achievable DL rates in multi-cell and singlecell systems versus $P_{\mathrm{D}}$ with $P_{\mathrm{T}}=3 \mathrm{dBm}$ and $\tau_{\mathrm{D}}=128$.

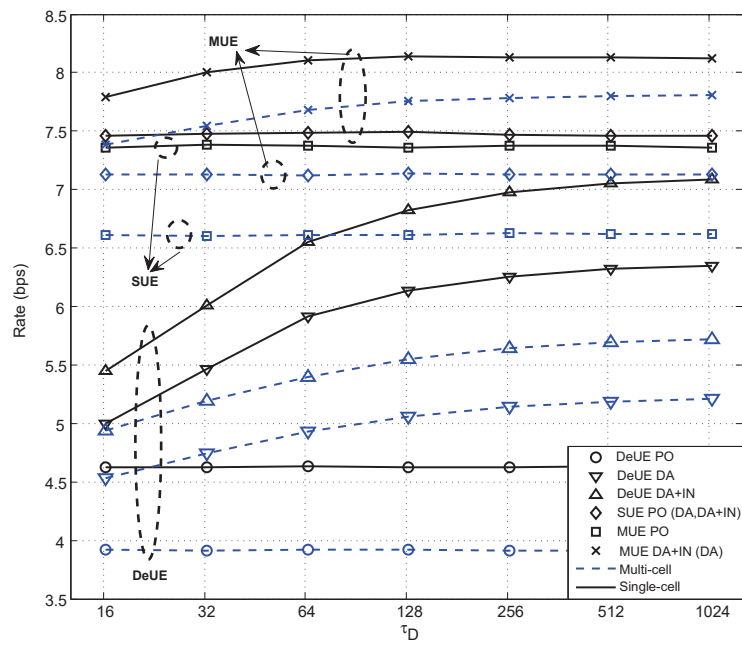

Fig. 9. Comparison of achievable DL rates in multi-cell and single-cell systems versus $\tau_{\mathrm{D}}$ with $P_{\mathrm{D}}=13 \mathrm{dBm}$ and $P_{\mathrm{T}}=3 \mathrm{dBm}$.

estimated channels (when $\tau_{\mathrm{D}} \rightarrow \infty$ ), 5.3 bps is the maximum rate of DeUEs achieved by DA methods and the maximum rate achieved by large $P_{\mathrm{D}}$ is $5.1 \mathrm{bps}$, thus we can infer that pilot contamination only leads to $0.2 \mathrm{bps}$ performance degradation and the rest $0.5 \mathrm{bps}$ rate gain loss ( totally $0.7 \mathrm{bps}$ from $1.8 \mathrm{bps}$ to $1.1 \mathrm{bps}$ ) is caused by inter-cell interference. In a similar way, we can infer that the $0.3 \mathrm{bps}$ decline of the rate gain from $\mathrm{ZF}$ IN is also caused by inter-cell interference.

\section{CONCLUSION}

In this paper, we developed a transmission scheme employing the DA MMSE channel estimator and ZF IN precoding for multi-cell HetNets with decoupling access to enhance the DL performance of DeUEs. Analytical approximations of the NMSE and achievable DL rate are derived under the proposed analytical framework, which enables us to compare NMSE and DL rate with or without DA methods as well as in single-cell and multi-cell systems. Analytical and numerical results show that: (a) MMSE DA outperforms conventional PO channel estimators with pilot contamination, (b) DA methods can boost DL rates of both DeUEs and MUEs by increasing UL data 
power and length, and (c) ZF IN can further improve DL rate of DeUEs by nulling the nearest interference without any cooperation and message transmission. In addition, inter-cell interference may cause more rate gain loss than pilot contamination using the proposed scheme in multi-cell HetNets.

\section{APPENDIX A \\ PROOF OF THEOREM 1}

Proof: We first recall the BER expression of binary modulations which can be expressed as [23]

$$
\mathrm{BER}=\int_{0}^{\infty} f_{\mathrm{SINR}}(x) \int_{\sqrt{x}}^{\infty} \frac{1}{\sqrt{2 \pi}} \exp \left(-\frac{1}{2} t^{2}\right) \mathrm{d} t \mathrm{~d} x
$$

where $f_{\text {SINR }}$ is the p.d.f. of the SINR. However, the exact distribution of SINR using MMSE detection is too complicated to obtain a closed-form expression of BER. Thus, the distribution of SINR is approximated by the well-defined Gamma distribution with p.d.f. shown as [19]

$$
f_{\text {Gam }}(x ; \alpha, \xi)=\frac{x^{\alpha-1} e^{-\frac{x}{\xi}}}{\Gamma(\alpha) \xi^{\alpha}} .
$$

To determine the two parameters $\alpha, \xi$ of Gamma distribution, moment matching and random matrix theory are utilized. Let $\mathrm{SINR}_{i k v}$ denote the SINR of the link from $\mathrm{UE}_{i k}$ to the $v$-th SBS in cell $i$. From [19], $\operatorname{SINR}_{i k v}$ can be written as

$$
\operatorname{SINR}_{i k v}=\frac{1}{\left[\left(\mathbf{I}_{K}+\rho_{i k v} \hat{\mathbf{G}}_{i i v}^{\mathrm{H}} \hat{\mathbf{G}}_{i i v}\right)^{-1}\right]_{k k}}-1,
$$

and following the approach in [11], $\operatorname{SINR}_{i k v}$ can be further simplified as

$$
\operatorname{SINR}_{i k v}=\rho_{i k v} \sum_{n=1}^{N} \frac{\left\|\varphi_{n}\right\|_{2}^{2}}{1+\rho_{i k v} d_{n}^{2}}
$$

where $\varphi_{n}$ follows i.i.d. $\mathcal{C N}\left(0, \hat{\beta}_{i j i v}^{\mathrm{S}}\right) ; d_{n}$ is the $n$-th eigenvalue of $\hat{\mathbf{G}}_{i i v(-k)}$ which is created by removing the $k$ th column of $\hat{\mathbf{G}}_{i \text { iv }}$. Define now an auxiliary matrix $\mathbf{A}=$ $\rho_{i k v} \hat{\mathbf{G}}_{i i v(-k)} \hat{\mathbf{G}}_{i i v(-k)}^{\mathrm{H}}$, therefore, we have

$$
\begin{aligned}
\frac{\operatorname{Tr}\left[\left(\mathbf{A}+\mathbf{I}_{N}\right)\right]}{N}= & \frac{1}{N} \sum_{n=1}^{N} \frac{1}{1+\rho_{i k v} d_{n}^{2}} \\
\stackrel{(a)}{=} & \int \frac{1}{1+x} \mathrm{~d} F^{\mathbf{A}}(x) \stackrel{(b)}{=} m_{\mathbf{A}}(-1) \triangleq \mu_{i k v}, \\
\frac{\operatorname{Tr}\left[\left(\mathbf{A}+\mathbf{I}_{N}\right)^{2}\right]}{N} & =\frac{1}{N} \sum_{n=1}^{N} \frac{1}{\left(1+\rho_{i k v} d_{n}^{2}\right)^{2}} \\
& \stackrel{(c)}{=} \int \frac{1}{(1+x)^{2}} \mathrm{~d} F^{\mathbf{A}}(x) \stackrel{(d)}{=} m^{\prime} \mathbf{A}(-1) \triangleq \sigma_{i k v}^{2},
\end{aligned}
$$

where $(b),(d)$ use the definition of the Stieltjes transform [28] and $m_{\mathbf{A}}(-1), m_{\mathbf{A}}^{\prime}(-1)$ are the Stieltjes transform of A and its derivative at -1 . Note that although $(a),(c)$ hold when $N$ is large, it is shown in [23] and simulations that this approximation is accurate even for very small dimensions.

By rewriting $\mathbf{A}=\frac{1}{N} \overline{\mathbf{G}}_{i i v(-k)} \boldsymbol{\Pi}_{i k v} \overline{\mathbf{G}}_{i i v(-k)}^{\mathrm{H}}, \boldsymbol{\Pi}_{i k v}$ is the diagonal matrix with $\left[\boldsymbol{\Pi}_{i k v}\right]_{j j}=\varpi_{i k v j}=N \rho_{i k v} \hat{\beta}_{i j i v}^{\mathrm{S}}$, and $\mathbb{E}\left[\overline{\mathbf{G}}_{i i v(-k)} \overline{\mathbf{G}}_{i i v(-k)}^{\mathrm{H}}\right]=\mathbf{I}_{N}$, we have [24]

$$
m_{\mathbf{A}}(z)=-\left(z-c \int \frac{\varpi \mathrm{d} F^{\Pi_{i k v}}(\varpi)}{1+\varpi m_{\mathbf{A}}(z)}\right)^{-1},
$$

and with some algebra manipulations the above equation is rewritten as

$$
m_{\mathbf{A}}(z)=-z m_{\mathbf{A}}^{2}(z)+c m_{\mathbf{A}}(z)-c m_{\Pi_{i k v}}\left(-\frac{1}{m_{\mathbf{A}}(z)}\right) .
$$

As $\Pi_{i k v}$ has real eigenvalues, (60) can be expressed as [24]

$$
m_{\mathbf{A}}(z)=\frac{c-1}{z}-\frac{c}{z} \frac{1}{K-1} \sum_{j=1}^{K-1} \frac{1}{m_{\mathbf{A}}(z) \varpi_{i k v j}+1},
$$

and taking the derivative on both sides of (61), we have

$$
\begin{aligned}
m_{\mathbf{A}}^{\prime}(z)=1-c- & \frac{c}{K-1} \sum_{j=1}^{K-1} \frac{\varpi_{i k v j} m^{\prime} \mathbf{A}(z)}{\left(m_{\mathbf{A}}(z) \varpi_{i k v j}+1\right)^{2}} \\
& +\frac{c}{K-1} \sum_{j=1}^{K-1} \frac{1}{m_{\mathbf{A}}(z) \varpi_{i k v j}+1} .
\end{aligned}
$$

Let $z=-1$ in (61), (62), we have (32) (33) and $\mu_{i k v}, \sigma_{i k v}^{2}$ are obtained by solving two equations numerically.

With the help of Lemma 1 in [11], the first two moments of $\mathrm{SINR}_{i k v}$ are given as

$$
\begin{aligned}
\mathbb{E}\left[\mathrm{SINR}_{i k v}\right] & \approx N \hat{\beta}_{i k i v}^{\mathrm{S}} \rho_{i k v} \mu_{i k v} \\
\operatorname{Var}\left[\mathrm{SINR}_{i k v}\right] & \approx N\left(\rho_{i k v} \hat{\beta}_{i k i v}^{\mathrm{S}}\right)^{2} \sigma_{i k v}^{2} .
\end{aligned}
$$

By plugging (63) (64) (54) into (53), the approximated closed-form BER expression is obtained.

\section{APPENDIX B}

PROOF OF THEOREM 2

Proof: Recalling the definition in (20) and (28), the problem is written

$$
\min _{\mathbf{C}_{i k i}^{\mathrm{TD}}} \mathbb{E}\left[\left\|\left(\mathbf{H}_{i i}\left(\hat{\mathbf{W}}_{i} \circ \mathbf{E}_{i}\right)+\sum_{l=1, l \neq i}^{L} \mathbf{H}_{l i} \mathbf{W}_{l}+\mathbf{Z}_{i 0}^{\mathrm{TD}}\right) \mathbf{C}_{i k i}^{\mathrm{TD}}-\mathbf{h}_{i k i}\right\|_{2}^{2}\right]_{(65)}
$$

and by expanding and differentiating (65) w.r.t. $\mathbf{C}_{i k i}^{\mathrm{TD}}$, we have

$$
\begin{array}{r}
\mathbb{E}\left[\left(\hat{\mathbf{W}}_{i} \circ \mathbf{E}_{i}\right)^{\mathrm{H}} \mathbf{H}_{i i}^{\mathrm{H}} \mathbf{H}_{i i}\left(\hat{\mathbf{W}}_{i} \circ \mathbf{E}_{i}\right)+\sum_{l=1, l \neq i}^{L} \mathbf{W}_{l}^{\mathrm{H}} \mathbf{H}_{l i}^{\mathrm{H}} \mathbf{H}_{l i} \mathbf{W}_{l}+\left(\mathbf{Z}_{i 0}^{\mathrm{TD}}\right)^{\mathrm{H}} \mathbf{Z}_{i 0}^{\mathrm{TD}}\right] \\
\times \mathbf{C}_{i k i}^{\mathrm{TD} *}=\mathbb{E}\left[\left(\hat{\mathbf{W}}_{i} \circ \mathbf{E}_{i}\right)^{\mathrm{H}} \mathbf{H}_{i i}^{\mathrm{H}} \mathbf{h}_{i k i}\right] .
\end{array}
$$

Considering the independency between channels and data sequences, and the prior information of error in (29) (30), the optimal solution of $\mathbf{C}_{i k i}^{\mathrm{TD}}$ in (34) (35) is obtained by following the similar approach as in [11].

Next, we will analyze the NMSE performance of DA MMSE. With the definition in (27) and (34), we can first calculate the numerator from (65) as

$$
\begin{gathered}
\mathbb{E}\left[\left\|\left(\mathbf{H}_{i i}\left(\hat{\mathbf{W}}_{i} \circ \mathbf{E}_{i}\right)+\sum_{l=1, l \neq i}^{L} \mathbf{H}_{l i} \mathbf{W}_{l}+\mathbf{Z}_{i 0}^{\mathrm{TD}}\right) \mathbf{C}_{i k i}^{\mathrm{TD}}-\mathbf{h}_{i k i}\right\|_{2}^{2}\right] \\
=M \beta_{i k i}^{\mathrm{M}}-M \beta_{i k i}^{\mathrm{M}}\left(\mathbf{C}_{i k i}^{\mathrm{TD}}\right)^{\mathrm{H}}\left(\hat{\mathbf{w}}_{i k} \circ \overline{\mathbf{e}}_{i k}\right)^{\mathrm{H}}-M \beta_{i k i}^{\mathrm{M}}\left(\hat{\mathbf{w}}_{i k} \circ \overline{\mathbf{e}}_{i k}\right) \mathbf{C}_{i k i}^{\mathrm{TD}} \\
+M\left(\mathbf{C}_{i k i}^{\mathrm{TD}}\right)^{\mathrm{H}}\left(\hat{\mathbf{P}}+\Delta \mathbf{P}+N_{0} \mathbf{I}\right)^{-1} \mathbf{C}_{i k i}^{\mathrm{TD}} \\
=M \beta_{i k i}^{\mathrm{M}}-M\left(\beta_{i k i}^{\mathrm{M}}\right)^{2}\left(\hat{\mathbf{w}}_{i k} \circ \overline{\mathbf{e}}_{i k}\right)\left(\hat{\mathbf{P}}+\Delta \mathbf{P}+N_{0} \mathbf{I}\right)^{-1}\left(\hat{\mathbf{w}}_{i k} \circ \overline{\mathbf{e}}_{i k}\right)^{\mathrm{H}}
\end{gathered}
$$


Then, define $\overline{\mathbf{W}}_{i}=\hat{\mathbf{W}}_{i} \circ \overline{\mathbf{E}}_{i}$ and the auxiliary matrix

$$
\mathbf{P}_{\text {Aux }}=\Delta \mathbf{P}+N_{0} \mathbf{I}=\left[\begin{array}{cc}
\mathbf{P}_{\mathrm{A}, 11} & \mathbf{0} \\
\mathbf{0} & \mathbf{P}_{\mathrm{A}, 22}
\end{array}\right],
$$

where

$$
\begin{aligned}
& \mathbf{P}_{\mathrm{A}, 11}=\mathbf{S}^{\mathrm{H}}\left(\sum_{l=1, l \neq i}^{L} \mathbf{R}_{\mathbf{H}_{l i}}\right) \mathbf{S}+N_{0} \mathbf{I}_{\tau_{\mathrm{T}}}, \\
& \mathbf{P}_{\mathrm{A}, 22}=p_{\mathrm{A}, 22} \mathbf{I}_{\tau_{\mathrm{D}}}
\end{aligned}
$$

and $p_{\mathrm{A}, 22}$ is shown in (40). Thus, we can write the second term of (67) without $M\left(\beta_{i k i}^{\mathrm{M}}\right)^{2}$ as

$$
\begin{aligned}
& \left(\hat{\mathbf{W}}_{i k} \circ \overline{\mathbf{e}}_{i k}\right)\left(\hat{\mathbf{P}}+\Delta \mathbf{P}+N_{0} \mathbf{I}\right)^{-1}\left(\hat{\mathbf{w}}_{i k} \circ \overline{\mathbf{e}}_{i k}\right)^{\mathrm{H}} \\
= & {\left[\overline{\mathbf{W}}_{i}\left(\overline{\mathbf{W}}_{i}^{\mathrm{H}} \mathbf{R}_{\mathbf{H}_{i i}} \overline{\mathbf{W}}_{i}+\mathbf{P}_{\text {Aux }}\right)^{-1} \overline{\mathbf{W}}_{i}^{\mathrm{H}}\right]_{k k} } \\
\stackrel{(a)}{=} & {\left[\overline{\mathbf{W}}_{i} \mathbf{P}_{\text {Aux }}^{-1} \overline{\mathbf{W}}_{i}^{\mathrm{H}}\right.} \\
& \left.-\overline{\mathbf{W}}_{i} \mathbf{P}_{\text {Aux }}^{-1} \overline{\mathbf{W}}_{i}^{\mathrm{H}}\left(\mathbf{R}_{\mathbf{H}_{i i}}^{-1}+\overline{\mathbf{W}}_{i} \mathbf{P}_{\text {Aux }}^{-1} \overline{\mathbf{W}}_{i}^{\mathrm{H}}\right)^{-1} \overline{\mathbf{W}}_{i} \mathbf{P}_{\text {Aux }}^{-1} \overline{\mathbf{W}}_{i}^{\mathrm{H}}\right]_{k k} \\
\stackrel{(b)}{=} & {\left[\mathbf{R}_{\mathbf{H}_{i i}}^{-1}-\mathbf{R}_{\mathbf{H}_{i i}}^{-1}\left(\overline{\mathbf{W}}_{i} \mathbf{P}_{\text {Aux }}^{-1} \overline{\mathbf{W}}_{i}^{\mathrm{H}}+\mathbf{R}_{\mathbf{H}_{i i}}^{-1}\right)^{-1} \mathbf{R}_{\mathbf{H}_{i i}}^{-1}\right]_{k k} }
\end{aligned}
$$

where $(a)$ uses the Woodbury matrix inversion identity and (b) follows from [29]

$$
\mathbf{A}-\mathbf{A}(\mathbf{A}+\mathbf{B})^{-1} \mathbf{A}=\mathbf{B}-\mathbf{B}(\mathbf{A}+\mathbf{B})^{-1} \mathbf{B} .
$$

By defining $\overline{\mathbf{E}}_{i}^{\mathrm{D}}=\mathbb{E}\left[\mathbf{E}_{i}^{\mathrm{D}}\right]$ and

$$
\tilde{\mathbf{E}}_{i}=\operatorname{diag}\left(\left(1-2 \mathrm{BER}_{i 1 v_{1}}\right), \ldots,\left(1-2 \mathrm{BER}_{i K v_{K}}\right)\right),
$$

the term inside the inverse of (71) can be further expanded as

$$
\begin{aligned}
& \overline{\mathbf{W}}_{i} \mathbf{P}_{\text {Aux }}^{-1} \overline{\mathbf{W}}_{i}^{\mathrm{H}} \\
& =\left[\mathbf{S}, \hat{\mathbf{X}}_{i} \circ \overline{\mathbf{E}}_{i}^{\mathrm{D}}\right]\left[\begin{array}{cc}
\mathbf{P}_{\mathrm{A}, 11}^{-1} & \mathbf{0} \\
\mathbf{0} & \mathbf{P}_{\mathrm{A}, 22}^{-1}
\end{array}\right]\left[\begin{array}{c}
\mathbf{S}^{\mathrm{H}} \\
\left(\hat{\mathbf{X}}_{i} \circ \overline{\mathbf{E}}_{i}^{\mathrm{D}}\right)^{\mathrm{H}}
\end{array}\right] \\
& =\mathbf{S P}_{\mathrm{A}, 11}^{-1} \mathbf{S}^{\mathrm{H}}+\frac{1}{p_{\mathrm{A}, 22}} \tilde{\mathbf{E}}_{i} \hat{\mathbf{X}}_{i} \hat{\mathbf{X}}_{i}^{\mathrm{H}} \tilde{\mathbf{E}}_{i}^{\mathrm{H}} \\
& \approx \rho^{\mathrm{PO}}\left(\mathbf{I}_{\tau_{\mathrm{T}}}-\left(\left(\rho^{\mathrm{PO}} \sum_{l=1, l \neq i}^{L} \mathbf{R}_{\mathbf{H}_{l i}}\right)^{-1}+\mathbf{I}_{\tau_{\mathrm{T}}}\right)^{-1}\right)+\frac{\tau_{\mathrm{D}} P_{\mathrm{D}}}{p_{\mathrm{A}, 22}} \tilde{\mathbf{E}}_{i}^{2} \\
& =\operatorname{diag}\left(\rho_{i 1}^{\mathrm{DA}, \mathrm{PC}}, \ldots, \rho_{i K}^{\mathrm{DA}, \mathrm{PC}}\right)
\end{aligned}
$$

where the approximation is from $\hat{\mathbf{X}} \hat{\mathbf{X}}^{\mathrm{H}} \rightarrow \tau_{\mathrm{D}} P_{\mathrm{D}} \mathbf{I}_{\tau_{\mathrm{D}}}$ which means that two decoded data sequences are uncorrelated when $\tau_{\mathrm{D}}$ is large.

Plugging (74) into (71) and then to (67), and with $\mathbb{E}\left[\left\|\mathbf{h}_{i k i}\right\|_{2}^{2}\right]=M \beta_{i k i}^{\mathrm{M}}$, the final result of NMSE in (38) is obtained.

\section{APPENDIX C}

PROOF OF LEMMA 1

Proof: From the results in (34), the estimated channel of $\mathrm{UE}_{i k}$ can be derived as

$$
\begin{aligned}
\hat{\mathbf{h}}_{i k i}^{\mathrm{DA}} & =\mathbf{Y}_{i 0}^{\mathrm{TD}}\left(\hat{\mathbf{P}}+\mathbf{P}_{\text {Aux }}\right)^{-1}\left(\hat{\mathbf{w}}_{i k} \circ \overline{\mathbf{e}}_{i k}\right)^{\mathrm{H}} \beta_{i k i}^{\mathrm{M}} \\
& =\mathbf{Y}_{i 0}^{\mathrm{TD}}\left[\left(\overline{\mathbf{W}}_{i}^{\mathrm{H}} \mathbf{R}_{\mathrm{H}_{i i}} \overline{\mathbf{W}}_{i}+\mathbf{P}_{\mathrm{Aux}}\right)^{-1} \overline{\mathbf{W}}_{i}^{\mathrm{H}}\right]_{k} \beta_{i k i}^{\mathrm{M}} \\
& \stackrel{(a)}{=} \mathbf{Y}_{i 0}^{\mathrm{TD}}\left[\mathbf{P}_{\text {Aux }}^{-1} \overline{\mathbf{W}}_{i}^{\mathrm{H}}\left(\overline{\mathbf{W}}_{i} \mathbf{P}_{\text {Aux }}^{-1} \overline{\mathbf{W}}_{i}^{\mathrm{H}}+\mathbf{R}_{\mathrm{H}_{i i}}^{-1}\right)^{-1} \mathbf{R}_{\mathrm{H}_{i i}}^{-1}\right]_{k} \beta_{i k i}^{\mathrm{M}} \\
& \stackrel{(b)}{\approx} \mathbf{Y}_{i 0}^{\mathrm{TD}}\left\{\frac{\beta_{i k i}^{\mathrm{M}}}{1+\beta_{i k i}^{\mathrm{M}} \rho_{k}^{\mathrm{DA}, \mathrm{PC}}} \mathbf{P}_{\text {Aux }}^{-1}\left(\hat{\mathbf{w}}_{i k} \circ \overline{\mathbf{e}}_{i k}\right)^{\mathrm{H}}\right\}
\end{aligned}
$$

where $(a)$ comes from [29]

$$
\left(\mathbf{P}^{-1}+\mathbf{B}^{\mathrm{H}} \mathbf{R}^{-1} \mathbf{B}\right)^{-1} \mathbf{B}^{\mathrm{H}} \mathbf{R}^{-1}=\mathbf{P B}^{\mathrm{H}}\left(\mathbf{B} \mathbf{P} \mathbf{B}^{\mathrm{H}}+\mathbf{R}\right)^{-1},
$$

and (b) follows the result in (74). By plugging the expressions of $\mathbf{Y}_{i 0}^{\mathrm{TD}}$ and $\mathbf{P}_{\text {Aux }}^{-1}$, we obtain

$$
\begin{aligned}
\hat{\mathbf{h}}_{i k i}^{\mathrm{DA}}= & \tilde{\beta}_{i k i}^{\mathrm{M}, \mathrm{DA}} \mathbf{H}_{i i}\left[\mathbf{S}, \hat{\mathbf{X}}_{i} \circ \mathbf{E}_{i}^{\mathrm{D}}\right]\left[\begin{array}{cc}
\mathbf{P}_{\mathrm{A}, 11}^{-1} & \mathbf{0} \\
\mathbf{0} & \mathbf{P}_{\mathrm{A}, 22}^{-1}
\end{array}\right]\left[\begin{array}{c}
\mathbf{s}_{k}^{\mathrm{H}} \\
\left(\hat{\mathbf{x}}_{i k} \circ \overline{\mathbf{e}}_{i k}^{\mathrm{D}}\right)^{\mathrm{H}}
\end{array}\right] \\
& +\tilde{\beta}_{i k i}^{\mathrm{M}, \mathrm{DA}}\left(\sum_{l=1, l \neq i}^{L} \mathbf{H}_{l i} \mathbf{W}_{l}+\mathbf{Z}_{i 0}^{\mathrm{TD}}\right) \mathbf{P}_{\mathrm{Aux}}^{-1}\left(\hat{\mathbf{w}}_{i k} \circ \overline{\mathbf{e}}_{i k}\right)^{\mathrm{H}} \\
= & \tilde{\beta}_{i k i}^{\mathrm{M}, \mathrm{DA}}\left(\rho_{k}^{\mathrm{PO}, \mathrm{PC}} \mathbf{h}_{i k i}+\frac{\mathbf{H}_{i i}}{p_{\mathrm{A}, 22}}\left[\begin{array}{c}
\boldsymbol{\chi}_{i 1} \overline{\boldsymbol{\chi}}_{i k}^{\mathrm{H}} \\
\vdots \\
\boldsymbol{\chi}_{i K} \overline{\boldsymbol{\chi}}_{i k}^{\mathrm{H}}
\end{array}\right]\right) \\
& +\tilde{\beta}_{i k i}^{\mathrm{M}, \mathrm{DA}} \sum_{l=1, l \neq i}^{L}\left(\rho_{k}^{\mathrm{PO}, \mathrm{PC}} \mathbf{h}_{l k i}+\frac{\mathbf{H}_{l i} \mathbf{X}_{l} \overline{\boldsymbol{\chi}}_{i k}^{\mathrm{H}}}{p_{\mathrm{A}, 22}}\right) \\
& +\frac{\rho_{i k}^{\mathrm{PO} \mathrm{PC}} \tilde{\beta}_{i k i}^{\mathrm{M}, \mathrm{DA}} \mathbf{N}_{i 0}^{\mathrm{UT}} \mathbf{s}_{k}^{\mathrm{H}}}{\tau_{\mathrm{T}} P_{\mathrm{T}}}+\frac{\tilde{\beta}_{i k i}^{\mathrm{M}, \mathrm{DA}} \mathbf{N}_{i 0}^{\mathrm{UD}} \overline{\boldsymbol{\chi}}_{i k}^{\mathrm{H}}}{p_{\mathrm{A}, 22}}
\end{aligned}
$$

then the result is achieved with some algebra manipulations.

From (43), it can be observed that $\hat{\mathbf{h}}_{i k i}^{\mathrm{DA}}$ is composed of independent Gaussian random vectors. Thus, $\hat{\mathbf{h}}_{i k i}^{\mathrm{DA}}$ is also Gaussian with zero mean, and its covariance matrix is calculated as

$$
\begin{aligned}
& \mathbb{E}\left[\hat{\mathbf{h}}_{i k i}^{\mathrm{DA}}\left(\hat{\mathbf{h}}_{i k i}^{\mathrm{DA}}\right)^{\mathrm{H}}\right] \\
& =\left(\tilde{\beta}_{i k i}^{\mathrm{M}, \mathrm{DA}}\right)^{2}\left\{\beta_{i k i}^{\mathrm{M}}\left(\left(\rho_{k}^{\mathrm{PO}, \mathrm{PC}}\right)^{2}+2 \rho_{k}^{\mathrm{PO}, \mathrm{PC}} \frac{\mathbb{E}\left[\boldsymbol{\chi}_{i k} \overline{\boldsymbol{\chi}}_{i k}^{\mathrm{H}}\right]}{p_{\mathrm{A}, 22}}+\frac{\mathbb{E}\left[\left(\boldsymbol{\chi}_{i k} \overline{\boldsymbol{\chi}}_{i k}^{\mathrm{H}}\right)^{2}\right]}{p_{\mathrm{A}, 22}^{2}}\right)\right. \\
& +\sum_{j \neq k} \beta_{i j i}^{\mathrm{M}} \frac{\mathbb{E}\left[\left(\chi_{i j} \bar{\chi}_{i k}^{\mathrm{H}}\right)^{2}\right]}{p_{\mathrm{A}, 22}^{2}}+\left(\rho_{k}^{\mathrm{PO}, \mathrm{PC}}\right)^{2}\left(\sum_{l=1, l \neq i}^{L} \beta_{l k i}^{\mathrm{M}}+\frac{1}{\rho_{k}^{\mathrm{PO}}}\right) \\
& \left.+\frac{\tau_{\mathrm{D}} P_{\mathrm{D}}\left(1-2 \mathrm{BER}_{i k i}\right)^{2}}{p_{\mathrm{A}, 22}^{2}}\left(P_{\mathrm{D}} \sum_{l=1, l \neq i}^{L} \sum_{j=1}^{K} \beta_{l j i}^{\mathrm{M}}+N_{0}\right)\right\} \mathbf{I}_{M} \\
& \stackrel{(a)}{=}\left(\tilde{\beta}_{i k i}^{\mathrm{M}, \mathrm{DA}}\right)^{2}\left\{\beta_{i k i}^{\mathrm{M}}\left(\rho_{k}^{\mathrm{DA}, \mathrm{PC}}\right)^{2}+\rho_{k}^{\mathrm{DA}, \mathrm{PC}}\right\} \mathbf{I}_{M}
\end{aligned}
$$

where $(a)$ is obtained with the result by using [30, Lemma 1]

$$
\mathbb{E}\left[\chi_{i k} \bar{\chi}_{i k}^{\mathrm{H}}\right]=\tau_{\mathrm{D}} P_{\mathrm{D}}\left(1-2 \mathrm{BER}_{i k v}\right)^{2},
$$

$\mathbb{E}\left[\left(\chi_{i j} \bar{\chi}_{i k}^{\mathrm{H}}\right)^{2}\right]=\tau_{\mathrm{D}} P_{\mathrm{D}}^{2}\left(1-\left(1-2 \mathrm{BER}_{i j v_{j}}\right)^{2}\right)\left(1-2 \mathrm{BER}_{i k v}\right)^{2}$.

The distribution of $\tilde{\mathbf{h}}_{i k i}^{\mathrm{DA}}$ can be derived by a similar approach.

\section{APPENDIX D}

Proof of THEOREM 3

Proof: Denote the effective channel $h_{i k i}^{\mathrm{Eff}} \triangleq \mathbb{E}\left[\mathbf{h}_{i k i}^{\mathrm{H}} \mathbf{v}_{i 0\left[k_{i}\right]}\right]$ and its expectation can be calculated as 


$$
\begin{aligned}
\mathbb{E}\left[h_{i k i}^{\mathrm{Eff}}\right] & =\mathbb{E}\left[\left(\hat{\mathbf{h}}_{i k i}^{\mathrm{DA}}\right)^{\mathrm{H}} \frac{\left.\hat{\mathbf{v}}_{i 0\left[k_{i}\right]}\right]}{\left\|\hat{\mathbf{v}}_{i 0\left[k_{i}\right]}\right\|}\right]=\mathbb{E}\left[\frac{1}{\left\|\hat{\mathbf{v}}_{i 0\left[k_{i}\right]}\right\|}\right]=\mathbb{E}\left[\sqrt{v_{i k}}\right] \\
& =\sqrt{\hat{\beta}_{i k i}^{\mathrm{M}, \mathrm{DA}}} \Upsilon\left(\frac{1}{2}, V_{i 0}\right)
\end{aligned}
$$

where $v_{i k}=\left(\left[\left(\left(\hat{\mathbf{H}}_{i}^{\mathrm{dl}}\right)^{\mathrm{H}} \hat{\mathbf{H}}_{i}^{\mathrm{dl}}\right)^{-1}\right]_{\left[k_{i}\right]\left[k_{i}\right]}\right)^{-1}$ and $v_{i k}$ is subject to Gamma $\left(V_{i 0}, \hat{\beta}_{i k i}^{\mathrm{M}, \mathrm{DA}}\right)$ [31]. Thus, the power of desired signal is calculated as

$$
\left|S_{i k}\right|^{2}=\frac{P_{\mathrm{M}}}{K_{i 0}^{\mathrm{dl}}}\left(\mathbb{E}\left[h_{i k i}^{\mathrm{Eff}}\right]\right)^{2}=\frac{P_{\mathrm{M}} \hat{\beta}_{i k i}^{\mathrm{M}, \mathrm{DA}}}{K_{i 0}^{\mathrm{dl}}} \Upsilon^{2}\left(\frac{1}{2}, V_{i 0}\right) .
$$

Then, the expectation of first interference power is given $\mathbb{E}\left[\left|I_{i k}^{(1)}\right|^{2}\right]=\frac{P_{\mathrm{M}}}{K_{i 0}^{\mathrm{dl}}} \operatorname{Var}\left[h_{i k i}^{\mathrm{Eff}}\right]=\frac{P_{\mathrm{M}}}{K_{i 0}^{\mathrm{d}}}\left(\mathbb{E}\left[\left(h_{i k i}^{\mathrm{Eff}}\right)^{2}\right]-\left(\mathbb{E}\left[h_{i k i}^{\mathrm{Eff}}\right]\right)^{2}\right)$,

where $\mathbb{E}\left[h_{i k i}^{\mathrm{Eff}}\right]$ is given in (79) and $\mathbb{E}\left[\left(h_{i k i}^{\mathrm{Eff}}\right)^{2}\right]$ is derived as

$$
\begin{aligned}
\mathbb{E}\left[\left(h_{i k i}^{\mathrm{Eff}}\right)^{2}\right] & =\mathbb{E}\left[v_{i k}\right]+\mathbf{v}_{i 0 k_{i}}^{\mathrm{H}} \mathbb{E}\left[\tilde{\mathbf{h}}_{i k i} \tilde{\mathbf{h}}_{i k i}^{\mathrm{H}}\right] \mathbf{v}_{i 0 k_{i}} \\
& =\hat{\beta}_{i k i}^{\mathrm{M}, \mathrm{DA}} V_{i 0}+\tilde{\beta}_{i k i}^{\mathrm{M}, \mathrm{DA}} .
\end{aligned}
$$

The power of interference due to channel estimation error is given as

$$
\begin{aligned}
\mathbb{E}\left[\left|I_{i k}^{(2)}\right|^{2}\right] & =\frac{P_{\mathrm{M}}}{K_{i 0}^{\mathrm{dl}}} \sum_{j \neq[k]} \mathbf{v}_{i 0 j}^{\mathrm{H}} \mathbb{E}\left[\tilde{\mathbf{h}}_{i k i} \tilde{\mathbf{h}}_{i k i}^{\mathrm{H}}\right] \mathbf{v}_{i 0 j} \\
& =\frac{P_{\mathrm{M}}\left(K_{i 0}^{\mathrm{dl}}-1\right)}{K_{i 0}^{\mathrm{dl}}} \tilde{\beta}_{i k i}^{\mathrm{M}, \mathrm{DA}} .
\end{aligned}
$$

Then, when $\mathrm{UE}_{l k}$ is served by the MBS in DL, we have $\hat{\mathbf{h}}_{i k l}^{\mathrm{MMSE}}=\frac{\beta_{k k l}^{\mathrm{M} l}}{\beta_{l k l}^{\mathrm{M}}} \hat{\mathbf{h}}_{l k l}^{\mathrm{MMSE}}$ from (10) and the interference power from the MBS of cell $l$ is derived as

$$
\begin{aligned}
& \mathbb{E}\left[\left|I_{i k, l}^{(3)}\right|^{2}\right]= \frac{P_{\mathrm{M}}}{K_{l 0}^{\mathrm{dl}}} \sum_{j=1}^{K_{l 0}^{\mathrm{dl}}}\left(\left(\frac{\beta_{i k l}^{\mathrm{M}}}{\beta_{l k l}^{\mathrm{M}}}\right)^{2} \mathbb{E}\left[\left|\left(\hat{\mathbf{h}}_{l k l}^{\mathrm{MMSE}}\right)^{\mathrm{H}} \mathbf{v}_{l 0 j}\right|^{2}\right]\right. \\
&\left.+\mathbb{E}\left[\left|\left(\tilde{\mathbf{h}}_{i k l}^{\mathrm{MMSE}}\right)^{\mathrm{H}} \mathbf{v}_{l 0 j}\right|^{2}\right]\right) \\
&=\frac{\left(M-K_{l 0}^{\mathrm{dl}}+1\right)^{\mathrm{dl}}}{K_{l 0}^{\mathrm{dl}}} P_{\mathrm{M}} \hat{\beta}_{i k l}^{\mathrm{M}}+P_{\mathrm{M}} \tilde{\beta}_{i k l}^{\mathrm{M}}
\end{aligned}
$$

and if $\mathrm{UE}_{l k}$ is served by a SBS, we have

$$
\mathbb{E}\left[\left|I_{i k, l}^{(3)}\right|^{2}\right]=\sum_{j=1}^{K_{l 0}^{\mathrm{dl}}} \frac{P_{\mathrm{M}}}{K_{l 0}^{\mathrm{dl}}} \mathbb{E}\left[\left|\mathbf{h}_{i k l}^{\mathrm{H}} \mathbf{v}_{l 0 j}\right|^{2}\right]=P_{\mathrm{M}} \beta_{i k l}^{\mathrm{M}} .
$$

Next, the power of interference from the $v$-th SBS which is the UL BS of $\mathrm{UE}_{i k}$ is derived as

$$
\mathbb{E}\left[\left|I_{i k}^{(4)}\right|^{2}\right]=\xi_{i v} \frac{P_{\mathrm{S}}^{\mathrm{dl}}}{K_{i v}^{\mathrm{dl}}} \sum_{j=1}^{\mathrm{dl}} \mathbb{E}\left[\left|\mathbf{v}_{i v j}^{\mathrm{H}} \tilde{\mathbf{g}}_{i k i v}^{\mathrm{MMSE}}\right|^{2}\right]=\xi_{i v} P_{\mathrm{S}} \tilde{\beta}_{i k i v}^{\mathrm{S}}
$$

where $\mathbb{E}\left[\left|\mathbf{v}_{i v j}^{\mathrm{H}} \hat{\mathbf{g}}_{i k i v}^{\mathrm{MMSE}}\right|^{2}\right]$ is forced to be 0 with the help of ZF IN precoding.

The power of interference from other SBSs in cell $i$ is

$$
\mathbb{E}\left[\left|I_{i k}^{(5)}\right|^{2}\right]=\sum_{s \neq v} \xi_{i s} \frac{P_{\mathrm{S}}}{K_{i s}^{\mathrm{d}}} \sum_{j=1}^{K_{i s}^{\mathrm{dl}}} \mathbb{E}\left[\left|\mathbf{g}_{i k i s}^{\mathrm{H}} \mathbf{v}_{i s j}\right|^{2}\right]=\sum_{s \neq v} \xi_{i s} P_{\mathrm{S}} \beta_{i k i s}^{\mathrm{S}} .
$$

Similar to the method in (84), the power of interference from the $s$-th SBS in cell $l$ is derived as when $\mathrm{UE}_{l k}$ is served by this SBS in UL/DL

$$
\begin{aligned}
\mathbb{E}\left[\left|I_{i k, l s}^{(6)}\right|^{2}\right] & =\xi_{l s} \frac{P_{\mathrm{S}}}{K_{l s}^{\mathrm{dl}}} \sum_{j=1}^{K_{l s}^{\mathrm{dl}}} \mathbb{E}\left[\left|\left(\frac{\beta_{i k l s}^{\mathrm{S}}}{\beta_{l k l s}^{\mathrm{S}}} \hat{\mathbf{g}}_{l k l s}^{\mathrm{MMSE}}+\tilde{\mathbf{g}}_{i k l s}^{\mathrm{MMSE}}\right)^{\mathrm{H}} \mathbf{v}_{l s j}\right|^{2}\right] \\
& =\xi_{l s} P_{\mathrm{S}}\left(\frac{\left(N-K_{l s}^{\mathrm{dl}}+1\right)}{K_{l s}^{\mathrm{dl}}} \hat{\beta}_{i k l s}^{\mathrm{S}}+\tilde{\beta}_{i k l s}^{\mathrm{S}}\right)
\end{aligned}
$$

and if $\mathrm{UE}_{l k}$ is a decoupled UE served by the $s$-th SBS in UL,

$$
\begin{aligned}
\mathbb{E}\left[\left|I_{i k, l s}^{(6)}\right|^{2}\right] & =\xi_{l s} \frac{P_{\mathrm{S}}}{K_{l s}^{\mathrm{dl}}} \sum_{j=1}^{K_{l s}^{\mathrm{dl}}} \mathbb{E}\left[\left|\left(\frac{\beta_{i k l s}^{\mathrm{S}}}{\beta_{l k l s}^{\mathrm{S}}} \hat{\mathbf{g}}_{l k l s}^{\mathrm{MMSE}}+\tilde{\mathbf{g}}_{i k l s}^{\mathrm{MMSE}}\right)^{\mathrm{H}} \mathbf{v}_{l s j}\right|^{2}\right] \\
& =\xi_{l s} \frac{P_{\mathrm{S}}}{K_{l s}^{\mathrm{dl}}} \sum_{j=1}^{\mathrm{dl}} \mathbb{E}\left[\left|\left(\tilde{\mathbf{g}}_{i k l s}^{\mathrm{MMSE}}\right)^{\mathrm{H}} \mathbf{v}_{l s j}\right|^{2}\right]=\xi_{l s} P_{\mathrm{S}} \tilde{\beta}_{i k l s}^{\mathrm{S}}
\end{aligned}
$$

and if $\mathrm{UE}_{l k}$ is a MUE, we obtain

$$
\mathbb{E}\left[\left|I_{i k, l s}^{(6)}\right|^{2}\right]=\xi_{l s} \frac{P_{\mathrm{S}}}{K_{l s}^{\mathrm{dl}}} \sum_{j=1}^{K_{l s}^{\mathrm{dl}}} \mathbb{E}\left[\left|\mathbf{g}_{i k l s}^{\mathrm{H}} \mathbf{v}_{l s j}\right|^{2}\right]=\xi_{l s} P_{\mathrm{S}} \beta_{i k l s}^{\mathrm{S}} .
$$

Finally, by plugging (80), (81), (83)-(90) into (49), the achievable rate in (48) is obtained.

\section{REFERENCES}

[1] J. G. Andrews, S. Buzzi, W. Choi, S. V. Hanly, A. Lozano, A. C. Soong, and J. C. Zhang, "What will 5G be?" IEEE J. Sel. Areas Commun., vol. 32, no. 6, pp. 1065-1082, Jun. 2014.

[2] F. Boccardi, R. W. Heath, Jr., A. Lozano, T. L. Marzetta, and P. Popovski, "Five disruptive technology directions for 5G," IEEE Commun. Mag., vol. 52, no. 2, pp. 74-80, Feb. 2014.

[3] J. G. Andrews, "Seven ways that HetNets are a cellular paradigm shift," IEEE Commun. Mag., vol. 51, no. 3, pp. 136-144, Mar. 2013.

[4] C.-X. Wang, F. Haider, X. Gao, X.-H. You, Y. Yang, D. Yuan, H. M. Aggoune, H. Haas, S. Fletcher, and E. Hepsaydir, "Cellular architecture and key technologies for $5 \mathrm{G}$ wireless communication networks," IEEE Commun. Mag., vol. 52, no. 2, pp. 122-130, Feb. 2014.

[5] L. Lu, G. Y. Li, A. L. Swindlehurst, A. Ashikhmin, and R. Zhang, "An overview of massive MIMO: Benefits and challenges," IEEE J. Sel. Topics Signal Process., vol. 8, no. 5, pp. 742-758, Oct. 2014.

[6] A. Adhikary, H. S. Dhillon, and G. Caire, "Massive-MIMO meets HetNet: Interference coordination through spatial blanking," IEEE J. Sel. Areas Commun., vol. 33, no. 6, pp. 1171-1186, Jun. 2015.

[7] S. Singh, X. Zhang, and J. G. Andrews, "Joint rate and SINR coverage analysis for decoupled uplink-downlink biased cell associations in HetNets," IEEE Trans. Wireless Commun., vol. 14, no. 10, pp. 53605373, Oct. 2015.

[8] J. Tang, D. K. So, E. Alsusa, K. A. Hamdi, and A. Shojaeifard, "Resource allocation for energy efficiency optimization in heterogeneous networks," IEEE J. Sel. Areas Commun., vol. 33, no. 10, pp. 2104-2117, Oct. 2015

[9] K. Smiljkovikj, P. Popovski, and L. Gavrilovska, "Analysis of the decoupled access for downlink and uplink in wireless heterogeneous networks," IEEE Wireless Commun. Lett., vol. 4, no. 2, pp. 173-176, Apr. 2015

[10] F. Boccardi, J. Andrews, H. Elshaer, M. Dohler, S. Parkvall, P. Popovski, and S. Singh, "Why to decouple the uplink and downlink in cellular networks and how to do it," IEEE Commun. Mag., vol. 54, no. 3, pp. 110-117, Mar. 2016.

[11] W. Liu, K.-K. Wong, S. Jin, and X. You, "A data-aided channel estimation scheme for decoupled systems in heterogeneous networks," IEEE Trans. Wireless Commun., vol. 17, no. 8, pp. 4987-5000, Aug. 2018.

[12] H. Elshaer, M. N. Kulkarni, F. Boccardi, J. G. Andrews, and M. Dohler, "Downlink and uplink cell association with traditional macrocells and millimeter wave small cells," IEEE Trans. Wireless Commun., vol. 15, no. 9, pp. 6244-6258, Sep. 2016. 
[13] M. Shi, K. Yang, C. Xing, and R. Fan, "Decoupled heterogeneous networks with millimeter wave small cells," IEEE Trans. Wireless Commun., vol. 17, no. 9, pp. 5871-5884, Sep. 2018.

[14] M. Bacha, Y. Wu, and B. Clerckx, "Downlink and uplink decoupling in two-tier heterogeneous networks with multi- antenna base stations," IEEE Trans. Wireless Commun., vol. 16, no. 5, pp. 2760-2775, May 2017.

[15] F. Fernandes, A. Ashikhmin, and T. L. Marzetta, "Inter-cell interference in noncooperative TDD large scale antenna systems," IEEE J. Sel. Areas Commun., vol. 31, no. 2, pp. 192-201, Feb. 2013.

[16] H. Yin, D. Gesbert, M. Filippou, and Y. Liu, "A coordinated approach to channel estimation in large-scale multiple-antenna systems," IEEE J. Sel. Areas Commun., vol. 31, no. 2, pp. 264-273, Feb. 2013.

[17] A. Ashikhmin and T. Marzetta, "Pilot contamination precoding in multicell large scale antenna systems," in Proc. IEEE Int. Symp. Inf. Theory (ISIT), Jul. 2012, pp. 1137-1141.

[18] J. Ma and P. Li, "Data-aided channel estimation in large antenna systems," IEEE Trans. Signal Process., vol. 62, no. 12, pp. 3111-3124, Jun. 2014

[19] H. Q. Ngo, E. G. Larsson, and T. L. Marzetta, "Energy and spectral efficiency of very large multiuser MIMO systems," IEEE Trans. Commun. vol. 61, no. 4, pp. 1436-1449, Apr. 2013.

[20] S. M. Kay, Fundamentals of Statistical Signal Processing: Estimation Theory. Upper Saddle River, NJ, USA: Prentice-Hall, Inc., 1993.

[21] X. Li, E. Björnson, E. G. Larsson, S. Zhou, and J. Wang, "Massive MIMO with multi-cell MMSE processing: Exploiting all pilots for interference suppression," EURASIP Journal on Wireless Communications and Networking, vol. 2017, no. 1, p. 117, Jun. 2017.

[22] Y. G. Li, J. H. Winters, and N. R. Sollenberger, "MIMO-OFDM for wireless communications: Signal detection with enhanced channel estimation," IEEE Trans. Commun., vol. 50, no. 9, pp. 1471-1477, Sep. 2002.

[23] P. Li, D. Paul, R. Narasimhan, and J. Cioffi, "On the distribution of SINR for the MMSE MIMO receiver and performance analysis," IEEE Trans. Inf. Theory, vol. 52, no. 1, pp. 271-286, Jan. 2006.

[24] J. W. Silverstein, "Strong convergence of the empirical distribution of eigenvalues of large dimensional random matrices," Journal of Multivariate Analysis, vol. 55, no. 2, pp. 331-339, Nov. 1995.

[25] G. Caire, N. Jindal, M. Kobayashi, and N. Ravindran, "Multiuser MIMO achievable rates with downlink training and channel state feedback," IEEE Trans. Inf. Theory, vol. 56, no. 6, pp. 2845-2866, Jun. 2010.

[26] A. Khansefid and H. Minn, "Achievable downlink rates of MRC and ZF precoders in massive MIMO with uplink and downlink pilot contamination," IEEE Trans. Commun., vol. 63, no. 12, pp. 4849-4864, Dec. 2015.

[27] J. Jose, A. Ashikhmin, T. L. Marzetta, and S. Vishwanath, "Pilot contamination and precoding in multi-cell TDD systems," IEEE Trans. Wireless Commun., vol. 10, no. 8, pp. 2640-2651, Aug. 2011.

[28] Z. Bai and J. W. Silverstein, Spectral Analysis of Large Dimensional Random Matrices. New York: Springer, 2010, vol. 20.

[29] G. Strang, Introduction to Linear Algebra, 4th ed. Wellesley, MA: Wellesley-Cambridge Press, 2009.

[30] W. Liu, S. Jin, and X. You, "On the downlink performance of decoupled HetNets with data-aided channel estimation," in Proc. IEEE Wireless Commun. Netw. Conf. (WCNC), Mar. 2019, pp. 2052-2057.

[31] D. K. Nagar and A. K. Gupta, "Expectations of functions of complex Wishart matrix," Acta applicandae mathematicae, vol. 113, no. 3, pp. 265-288, Mar. 2011.

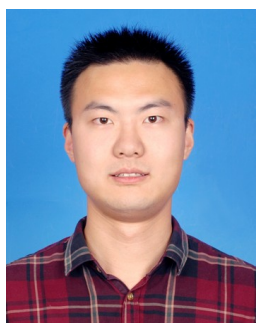

Wen Liu received the B.S. degree in information engineering from Southeast University, Nanjing, China, in 2013. He is currently pursuing the Ph.D. degree in the School of Information Science and Engineering at Southeast University. From 2016 to 2018, he was a Visiting Student with the Department of Electrical Engineering, University of Notre Dame, Notre Dame, IN, USA. His research interests include massive MIMO wireless communications, channel estimation, stochastic geometry and and its applications in heterogeneous networks.

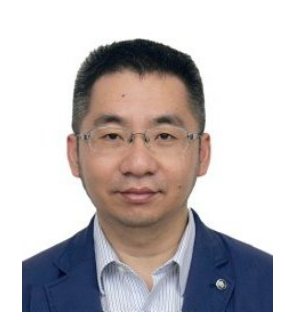

Shi Jin (S'06-M'07-SM'17) received the B.S. degree in communications engineering from Guilin University of Electronic Technology, Guilin, China, in 1996, the M.S. degree from Nanjing University of Posts and Telecommunications, Nanjing, China, in 2003, and the Ph.D. degree in information and communications engineering from the Southeast University, Nanjing, in 2007. From June 2007 to October 2009, he was a Research Fellow with the Adastral Park Research Campus, University College London, London, U.K. He is currently with the faculty of the National Mobile Communications Research Laboratory, Southeast University. His research interests include space time wireless communications, random matrix theory, and information theory. He serves as an Associate Editor for the IEEE Transactions on Wireless Communications, and IEEE Communications Letters, and IET Communications. Dr. Jin and his co-authors have been awarded the 2011 IEEE Communications Society Stephen O. Rice Prize Paper Award in the field of communication theory and a 2010 Young Author Best Paper Award by the IEEE Signal Processing Society.

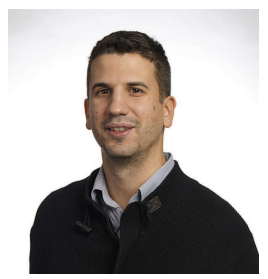

Michail Matthaiou (S'05-M'08-SM'13) was born in Thessaloniki, Greece in 1981. He obtained the Diploma degree (5 years) in Electrical and Computer Engineering from the Aristotle University of Thessaloniki, Greece in 2004. He then received the M.Sc. (with distinction) in Communication Systems and Signal Processing from the University of Bristol, U.K. and Ph.D. degrees from the University of Edinburgh, U.K. in 2005 and 2008, respectively. From September 2008 through May 2010, he was with the Institute for Circuit Theory and Signal Processing, Munich University of Technology (TUM), Germany working as a Postdoctoral Research Associate. He is currently a Reader (equivalent to Associate Professor) in Multiple-Antenna Systems at Queen's University Belfast, U.K. after holding an Assistant Professor position at Chalmers University of Technology, Sweden. His research interests span signal processing for wireless communications, massive MIMO systems, hardware-constrained communications, mm-wave systems and deep learning for communications.

Dr. Matthaiou and his coauthors received the IEEE Communications Society (ComSoc) Leonard G. Abraham Prize in 2017. He was awarded the prestigious 2018/2019 Royal Academy of Engineering/The Leverhulme Trust Senior Research Fellowship and recently received the 2019 EURASIP Early Career Award. His team was also the Grand Winner of the 2019 Mobile World Congress Challenge. He was the recipient of the 2011 IEEE ComSoc Best Young Researcher Award for the Europe, Middle East and Africa Region and a co-recipient of the 2006 IEEE Communications Chapter Project Prize for the best M.Sc. dissertation in the area of communications. He has co-authored papers that received best paper awards at the 2018 IEEE WCSP and 2014 IEEE ICC and was an Exemplary Reviewer for IEEE COMMUNICATIONS LETTERS for 2010.In 2014, he received the Research Fund for International Young Scientists from the National Natural Science Foundation of China.He is currently the Editor-in-Chief of Elsevier Physical Communication and a Senior Editor for IEEE WIRELESS COMMUNICATIONS LETTERS. In the past, he was an Associate Editor for the IEEE TRANSACTIONS ON COMMUNICATIONS and Associate Editor/Senior Editor for IEEE COMMUNICATIONS LETTERS. 


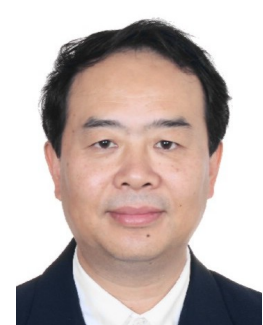

Xiao-Hu You was born in August 25, 1962. He received his Master and Ph.D. Degrees from Southeast University, Nanjing, China, in Electrical Engineering in 1985 and 1988, respectively. Since 1990, he has been working with National Mobile Communications Research Laboratory at Southeast University, where he holds the rank of director and professor. His research interests include mobile communication systems, signal processing and its applications. $\mathrm{He}$ has contributed over 100 IEEE journal papers and 2 books in the areas of adaptive signal processing, neural networks and their applications to communication systems. From 1999 to 2002, he was the Principal Expert of the C3G Project, responsible for organizing China's 3G Mobile Communications R\&D Activities. From 2001 to 2006, he was the Principal Expert of the China National 863 Beyond 3G FuTURE Project. Since 2013, he has been the Principal Investigator of China National 863 5G Project.

Professor You served as the general chairs of IEEE WCNC 2013 and IEEE VTC 2016. Now he is Secretary General of the FuTURE Forum, vice Chair of China IMT-2020 Promotion Group, vice Chair of China National Mega Project on New Generation Mobile Network. He was the recipient of the National 1st Class Invention Prize in 2011, and he was selected as IEEE Fellow in same year due to his contributions to development of mobile communications in China. 